\title{
Reassembly and co-crystallization of a family 9 processive endoglucanase from its component parts: Structural and functional significance of the intermodular linker
}

Svetlana Petkun, Inna Rozman Grinberg, Raphael Lamed, Sadanari Jindou, Tal Burstein, Oren Yaniv, Yuval Shoham, Linda J.W. Shimon, Edward A Bayer, Felix Frolow

Non-cellulosomal processive endoglucanase 9I (Cel9I) from Clostridium thermocellum is a modular protein, consisting of a family-9 glycoside hydrolase (GH9) catalytic module and two family-3 carbohydrate-binding modules (CBM3c and CBM3b), separated by linker regions. GH9 does not show cellulase activity when expressed without $\mathrm{CBM3C}$ and $\mathrm{CBM} 3 \mathrm{~b}$ and the presence of the $\mathrm{CBM} 3 \mathrm{C}$ was previously shown to be essential for endoglucanase activity. Physical reassociation of independently expressed GH9 and CBM3c modules (containing linker sequences) restored $60-70 \%$ of the intact Cel9l endocellulase activity. However, the mechanism responsible for recovery of activity remained unclear. In this work we independently expressed recombinant $\mathrm{GH} 9$ and $\mathrm{CBM3c}$ with and without their interconnecting linker in Escherichia coli. We crystallized and determined the molecular structure of the GH9/linker-CBM3c heterodimer at a resolution of $1.68 \AA$ to understand the functional and structural importance of the mutual spatial orientation of the modules and the role of the interconnecting linker during their re-association. Enzyme activity assays and isothermal titration calorimetry were performed to study and compare the effect of the linker on the re-association. The results indicated that reassembly of the modules could also occur without the linker, albeit with only very low recovery of endoglucanase activity. We propose that the linker regions in the GH9/CBM3c endoglucanases are important for spatial organization and fixation of the modules into functional enzymes. 


\section{Reassembly and co-crystallization of a family 9 processive}

2 endoglucanase from its component parts:

3 Structural and functional significance of the intermodular linker

5 Svetlana Petkun ${ }^{1}$, Inna Rozman Grinberg ${ }^{1}$, Raphael Lamed ${ }^{1}$, Sadanari Jindou², Tal

6 Burstein ${ }^{1}$, Oren Yaniv ${ }^{1}$, Yuval Shoham ${ }^{3}$, Linda J.W. Shimon ${ }^{4}$, Edward A. Bayer ${ }^{5, *}$, and

7 Felix Frolow ${ }^{1}$

8

$9{ }^{1}$ Department of Molecular Microbiology and Biotechnology, The Daniella Rich Institute for

10 Structural Biology, Tel Aviv University, Ramat Aviv 69978 ISRAEL

112 Department of Life Sciences, Meijo University, Nagoya 468-8502 JAPAN

$12{ }^{3}$ Department of Biotechnology and Food Engineering, Technion-Israel Institute of Technology,

13 Haifa 32000 ISRAEL

$14{ }^{4}$ Department of Chemical Research Support and

15 5epartment of Biological Chemistry, The Weizmann Institute of Science, Rehovot 76100

16 ISRAEL

Corresponding author:

Edward A. Bayer

Department of Biological Chemistry

The Weizmann Institute of Science

Rehovot 76100 ISRAEL

Tel: 972-8-934-2373

Fax: 972-8-946-8256

Email: ed.bayer@weizmann.ac.il

20 Running title: Crystal structure of reassembled Ce19I

21 Abbreviations used: CBM, carbohydrate-binding module; $C B M 3 c L$, family $3 \mathrm{c} C B M$ with

22 linker; $\mathrm{CBM} 3 \mathrm{c} N L$, family 3c $\mathrm{CBM}$ without linker; $\mathrm{CMC}$, carboxymethyl cellulose; GH9, family

239 glycoside hydrolase; ITC, isothermal titration calorimetry; PASC, phosphoric acid-swollen

24 cellulose; PEG, polyethylene glycol; SeMet, selenium-methionine labeled derivative. 


\section{Abstract.}

26 Non-cellulosomal processive endoglucanase 9I (Ce19I) from Clostridium thermocellum is a

27 modular protein, consisting of a family-9 glycoside hydrolase (GH9) catalytic module and two

28 family-3 carbohydrate-binding modules (CBM3c and CBM3b), separated by linker regions. GH9

29 does not show cellulase activity when expressed without CBM3c and CBM3b and the presence

30 of the CBM3c was previously shown to be essential for endoglucanase activity. Physical reassociation of independently expressed GH9 and CBM3c modules (containing linker sequences) restored $60-70 \%$ of the intact Cel9I endocellulase activity. However, the mechanism responsible for recovery of activity remained unclear. In this work we independently expressed recombinant GH9 and CBM3c with and without their interconnecting linker in Escherichia coli. We crystallized and determined the molecular structure of the GH9/linker-CBM3c heterodimer at a resolution of $1.68 \AA$ to understand the functional and structural importance of the mutual spatial orientation of the modules and the role of the interconnecting linker during their reassociation. Enzyme activity assays and isothermal titration calorimetry were performed to study and compare the effect of the linker on the re-association. The results indicated that reassembly of the modules could also occur without the linker, albeit with only very low recovery of endoglucanase activity. We propose that the linker regions in the GH9/CBM3c endoglucanases are important for spatial organization and fixation of the modules into functional enzymes. 
45

46

47

48

49

50

51

52

53

54

55

56

57

58

59

60

61

62

63

64

65

66

67

68

69

\section{Introduction}

Cellulose is a major component of the plant cell wall, lending structural stability and resilience to an otherwise flaccid material. The propensity of cellulose to form ordered, tightly packed, para-crystalline fibrils hinders its enzymatic degradation. Indeed, the recalcitrant properties of cellulose are such that numerous enzymes are required to act synergistically in achieving its efficient degradation. Many types of bacteria and fungi are capable of degrading cellulose and other plant cell wall polysaccharides in an effective manner, producing a variety of various cellulases and related enzymes, either existing in the free state, or associated with a multi-enzyme complex known as the cellulosome (Bayer et al. 2004; Bayer et al. 2008; Demain et al. 2005; Doi \& Kosugi 2004; Fontes \& Gilbert 2010). Clostridium thermocellum is an anaerobic thermophilic bacterium, known for its efficient degradation of cellulose and other plant cell wall polysaccharides (Béguin et al. 1992; Freier et al. 1988; Garcia-Martinez et al. 1980; Ng et al. 1977; Wiegel et al. 1985). The cellulase system of this bacterium includes a remarkable variety of enzymes, some existing in the free state but most associated with a highly efficient multi-enzyme complex, termed cellulosome (Bayer et al. 2004; Lamed et al. 1983a; Lamed et al. 1983b; Shoham et al. 1999), capable of converting a wide variety of plantderived polysaccharides directly into soluble sugars and fermentation products (Béguin \& Alzari 1998; Felix \& Ljungdahl 1993; Schwarz 2001; Schwarz et al. 2004). These capabilities render $C$. thermocellum a high utility candidate for use in consolidated bioprocessing (CBP) applications [reviewed in (Akinosho et al. 2014)].

Cellulases are a class of modular enzymes with a catalytic glycoside hydrolase $(\mathrm{GH})$ module that hydrolyzes the $\beta$-1,4-glucosidic bond of the cellulose chain (Cantarel et al. 2009; Davies \& Henrissat 1995; Gilbert \& Hazlewood 1993; Henrissat 1991; Henrissat \& Davies 1997; Wilson \& Irwin 1999). The catalytic module is usually associated with various numbers of accessory modules that serve to modulate the enzyme activity, and the enzymes have been categorized into families according to the amino-acid sequence of the GH domain (Cantarel et al. 2009; Gilkes et al. 1991; Henrissat \& Davies 1997; Henrissat \& Davies 2000; Henrissat \& Romeu 1995).

2 Cellulases have been broadly divided into two types: endoglucanases that can hydrolyze bonds internally in cellulose chain, and exoglucanases that act preferentially on chain ends, progressively cleaving off cellobiose as the main product. The distinction between endo- and 
exo-acting enzymes is also reflected by the architecture of the respective class of active site, whereby endoglucanases, for example, are commonly characterized by a groove or open binding cleft, into which any part of the linear cellulose chain can fit. On the other hand, the exoglucanases bear tunnel-like active sites, which can only accept a substrate chain via its terminus (either the reducing or non-reducing end, depending on the enzyme), thereby cleaving cellulose in a sequential manner. The sequential hydrolysis of a cellulose chain has earned the term "processivity" (Beckham et al. 2014; Davies \& Henrissat 1995; Wilson \& Kostylev 2012), and processive enzymes are considered to be key components which contribute to the overall efficiency of a given cellulase system. Some endoglucanases, notably from GH family 9 , have also been shown to sequentially hydrolyze cellulose chains and are thus referred to as processive endoglucanases (Gal et al. 1997; Gilad et al. 2003; Irwin et al. 1998; Jeon et al. 2012; Kuusk et al. 2015; Zverlov et al. 2003). Such enzymes appear to possess extended catalytic clefts and the observed processivity appears to require highly coordinated substrate-binding affinities from opposite sides of the cleavage site (Bu et al. 2012; Li et al. 2010; Payne et al. 2011).

Cellulase 9I (Ce19I), is a non-cellulosomal family 9 processive endoglucanase from Clostridium thermocellum, which degrades crystalline cellulose (Avicel and filter paper) as well as phosphoric acid-swollen cellulose (PASC) and carboxymethyl cellulose (CMC) (Gilad et al. 2003). This enzyme consists of a catalytic GH9 module at its $\mathrm{N}$ terminus, followed by two family 3 carbohydrate-binding modules (CBMs): CBM3c and CBM3b. The three modules are separated by distinctive linker sequences. Such intermodular linker segments were proposed to be important for the physical association of the modules in the space, and to promote intermodular and/or intersubunit protein-protein interactions (Bayer et al. 1998; Bayer et al. 2009; Noach et al. 2008).

\section{The C-terminal CBM3b module, as a classic CBM3, is responsible for targeting the Ce19I} enzyme to the planar surface of the crystalline cellulose substrate (Gilad et al. 2003; Su et al. 2012; Tormo et al. 1996). It has also been proposed to disrupt the crystalline regions of cellulose, rendering it more accessible to the GH9 catalytic module (Yi et al. 2013) and to contribute to enzyme processivity by preventing the desorption of the catalytic module from cellulose (Telke et al. 2012). The function of the CBM3c is less straightforward. Removal of CBM3c from $C$. thermocellum Ce19I and from C. cellulolyticum Ce19G. P. Barcinonensis Ce19B significantly reduces the enzyme activity (Burstein et al. 2009; Chiriac et al. 2010; Gal et al. 1997). CBM3c 
106 modules have been shown to alter the normal function of the GH9 catalytic module of

107 Thermobifida fusca Ce19A from the standard endo-acting mode into a processive endoglucanase

108 (Bayer et al. 1998; Irwin et al. 1998). Thus, Gilad et al.(Gilad et al. 2003) showed in 2003 that

109 the endoglucanase activity of Ce19I is dependent upon the presence of the CBM3c module and

110 suggested that the fused $\mathrm{CBM} 3 \mathrm{c}$ serves an important accessory role for the catalytic domain by

111 altering its character to facilitate processive cleavage of recalcitrant cellulose substrates.

112 In addition to the Cel9 CBM3c, several other examples of CBMs that are considered to

113 modulate catalytic specificity and act cooperatively with the catalytic domain have recently been

114 discovered. These include CBM66 that directs the cognate enzyme towards highly branched

115 glucans rather than linear fructose polymers (Cuskin et al. 2012), CBM48 that contributes to

116 substrate binding at the active site of a glucan phosphatase (Meekins et al. 2014), family-43 $\beta$ -

117 xylosidases where the GH43 is complemented by an additional module that confers hydrolytic

118 activity to the mature enzyme (Moraïs et al. 2012), and CBM46, that constitutes part of the

119 catalytic cleft required for the hydrolysis of $\beta-1,3-1,4$-glucans (Venditto et al. 2015). The

120 carbohydrate-binding PA14 domain is also known to affect substrate binding of the catalytic

121 domain by contributing to the formation of its active site (Gruninger et al. 2014; Zmudka et al.

122 2013).

123 We have previously shown that independently expressed GH9 and linker-containing CBM3c

124 modules of Ce19I readily re-associate in vitro and that this physical reassociation recovers 60-

$12570 \%$ of the intact Ce19I endoglucanase activity (Burstein et al. 2009).

126 We have examined in this work the interaction of the CBM3c with the catalytic module either

127 with or without the intermodular linker in order to better understand the function of the CBM3c

128 in the family-9 enzymes and the role of the linkers regions. The effect of the re-association of the

129 CBM3c with linker $(\mathrm{CBM} 3 \mathrm{c} L)$ and the $\mathrm{CBM} 3 \mathrm{c}$ without linker $(\mathrm{CBM} 3 \mathrm{c} N L)$ on the enzymatic

130 activity of GH9 has been studied by the crystallization and structure determination of the

131 reassembled GH9-CBM3c $L$ complex at a resolution of $1.68 \AA$. The results of this study will help

132 us to understand the contribution of ancillary modules in the action of multi-modular glycoside

133 hydrolases.

134 


\section{Materials and methods}

\section{Cloning of the GH9, CBM3CL and CBM3cNL proteins}

137 Cloning of the DNA fragments encoding the C-terminally His-tagged CBM3c with the linker 138 and the untagged GH9 module from Cel9I of C. thermocellum (GenBank accession code 139 L04735) was described earlier (Burstein et al. 2009; Gilad et al. 2003). C-terminally His-tagged

$140 \mathrm{CBM} 3 \mathrm{c}$ without the linker connecting it to the GH9 was amplified using the same procedure and 141 the following primers: $\mathrm{F}^{\prime}-5^{\prime}$ CCATGGGCGAAGTTCCGGAGGATGAAATA and $\mathrm{R}^{\prime}-$

1425 ' $5^{\prime}$ TCGAGCGGTtCCCTTCCAAATACCAG. The PCR products were purified and cleaved with 143 restriction enzymes $\mathrm{NcoI}$ and $\mathrm{XhoI}$ and inserted into the pET-28a(+) expression vector (Novagen, 144 Madison, WI, USA).

\section{Expression and purification of recombinant proteins}

146 The GH9 and CBM3c modules both with (GH9L, CBM3cL) and without (GH9NL and $147 \mathrm{CBM} 3 \mathrm{cNL}$ ) the linker regions were expressed independently by the identical expression 148 procedure. Escherichia coli strain BL21(DE3)RIL harboring the plasmids was aerated at $310 \mathrm{~K}$ 149 in 3-liters Terrific Broth supplemented with $25 \mathrm{mg} \mathrm{ml}^{-1}$ kanamycin. After $3 \mathrm{~h}$, the culture 150 reached an $\mathrm{A}_{600}$ of 0.6; $0.1 \mathrm{mM}$ isopropyl- $\beta$-D-1-thiogalactopyranoside was added to induce 151 gene expression, and cultivation was continued at $310 \mathrm{~K}$ for an additional $12 \mathrm{~h}$. Cells were 152 harvested by centrifugation $(5,000 \times \mathrm{g}$ for $15 \mathrm{~min})$ at $277 \mathrm{~K}$ and were subsequently re-suspended 153 in $50 \mathrm{mM} \mathrm{NaH}_{2} \mathrm{PO}_{4}, \mathrm{pH} 8.0$, containing $300 \mathrm{mM} \mathrm{NaCl}$ at a ratio of $1 \mathrm{~g}$ wet pellet to $4 \mathrm{ml}$ buffer 154 solution. A few micrograms of DNase powder were added prior to the sonication procedure. The 155 suspension was kept on ice during sonication, after which it was centrifuged $(20,000 \times \mathrm{g}$ at 277 $156 \mathrm{~K}$ for $20 \mathrm{~min}$ ), and the supernatant was collected.

157 The soluble expressed His-tagged CBM3c modules with or without the linker, according to 158 the type of the experiment, were applied batchwise to Ni-IDA resin during 1-h incubation with 159 gentle stirring at $4{ }^{\circ} \mathrm{C}$. Non-specifically bound proteins were washed with a buffer containing 50 $160 \mathrm{mM} \mathrm{NaH}_{2} \mathrm{PO}_{4} \mathrm{pH} 6,300 \mathrm{mM} \mathrm{NaCl}, 10 \%$ glycerol and $10 \mathrm{mM}$ imidazole. Crude extract 161 supernatant fluids, containing the expressed GH9 module, were added to the CBM3c-bound Ni162 IDA resin, and the mixture was incubated overnight with gentle stirring at $4{ }^{\circ} \mathrm{C}$. The adsorbed 163 protein complexes were eluted with $300 \mathrm{mM}$ imidazole and subjected to further purification by 
164 size-exclusion chromatography. Fast protein liquid chromatography (FPLC) was performed 165 using a Superdex 75pg column and ÄKTA Prime system (GE Healthcare, Piscataway, NJ) to 166 further purify the complex. One peak, corresponding approximately to $70 \mathrm{kDa}$, matching the

167 predicted molecular weight of the GH9-CBM3c complex, was observed in the chromatogram.

168 The 15 amino-acid linker sequence (about $1.5 \mathrm{kDa}$ ) did not significantly affect the elution

169 volume, compared to that of the complex without the linker, presumably due to the limited

170 resolution of the column. The relevant fractions (the purified complexed proteins) were analyzed

171 by $15 \%$ sodium dodecyl sulfate-polyacrylamide gel electrophoresis (SDS-PAGE) with

172 Coomassie brilliant blue staining. Two clear bands, of about 52 and $19.5 \mathrm{kDa}$ were observed.

173 The rearranged modules were concentrated to $6 \mathrm{mg} \mathrm{ml}^{-1}$ using Centriprep YM-3 centrifugal filter

174 devices YM-3 (Amicon Bioseparation, Millipore Corporation, Bedford, USA). Protein

175 concentration was determined by measuring UV absorbance at $280 \mathrm{~nm}$.

176 The full-length Ce19I was purified by affinity chromatography on Avicel as reported earlier

177 (Burstein et al. 2009; Gilad et al. 2003).

178

179

180

181

182

183

184

185

186

187

188

189

190

191

192

\section{Microcalorimetric analysis}

Isothermal titration calorimetry (ITC) experiments were carried out using a VP-ITC MicroCalorimeter (MicroCal, LLC, Northampton, MA) at $298 \mathrm{~K}$. About $300 \mu \mathrm{M}$ solution of CBM3c $N L$ was injected into a $65 \mu \mathrm{M}$ solution of GH9. The reaction was performed in a buffer containing $50 \mathrm{mM}$ Tris- $-\mathrm{HCl}, \mathrm{pH} 7.5,150 \mathrm{mM} \mathrm{NaCl}, 0.05 \%$ sodium azide. Heats of dilution of the titrants were subtracted from the titration data, and the corrected data were analyzed using the Origin ITC analysis software package supplied by MicroCal. Thermal titration data were fit to the one binding site model, and enthalpy $(\Delta \mathrm{H})$, entropy $(\Delta \mathrm{S})$, association constant $(\mathrm{Ka})$ and stoichiometry of binding $(\mathrm{N})$ were determined. In all cases, the calculated stoichiometry $(\mathrm{N})$ was lower than one, most likely due to the fact that the CBM3 proteins lost their native functionality with time. For the analysis, the CBM3 protein concentrations were corrected as to provide a stoichiometry of one. Two titrations were performed to evaluate reproducibility.

\section{Enzyme activity assay}

Reactions were performed at $333 \mathrm{~K}$, in $50 \mathrm{mM}$ citrate buffer (pH 6.0). The soluble cellulolytic substrate was carboxymethyl cellulose (CMC, Sigma Chem. Co. St. Louis, MO). The amount of 
193 reducing sugars released from the substrate was determined with the 3,5-dinitrosalicylic acid 194 (DNS) reagent as described by Miller et al (Miller 1959). Activity was defined as the amount 195 (micromole) of reducing sugar released after $10 \mathrm{~min}$ of reaction.

\section{Crystallization}

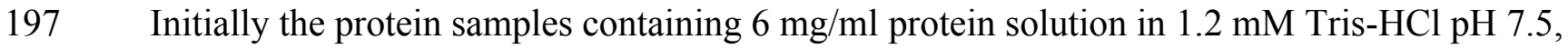
$1981.5 \mathrm{mM}$ sodium chloride, $0.025 \%$ sodium azide, were screened, using the microbatch 199 crystallization method under 1:1 mixture of silicon and paraffin oil (Chayen et al. 1990), using 200288 conditions from the Hampton Research HT screens (SaltRx, Index HT, and Crystal Screen 201 HT; Hampton Research, Aliso Viejo, CA) and 96 conditions of the Wizard Crystallization kit 202 from Emerald BioSystems (Rigaku Reagents, Bainbridge Island, WA). The dyad of GH9 and $203 \mathrm{CBM} 3 \mathrm{c} N L$ did not yield any crystals. Screening of the GH9-CBM3c $L$ resulted in plate-like 204 crystals that appeared after several days under several conditions, all of which contained PEG 2053350 and 4000. The best crystals were obtained in $30 \%$ PEG (both 3350 and 4000), $0.2 \mathrm{M}$ 206 magnesium chloride, and $0.1 \mathrm{M}$ Hepes, $\mathrm{pH}$ 7.5. Attempts to optimize this condition using microbatch, hanging-drop, and sitting drop methods were unsuccessful, as the crystals remained very thin and fragile. The superfine Eyelash (Ted Pella, Inc, Redding, CA) was used to touch these crystals and consequently to streak the sitting drops, composed of $5 \mu \mathrm{l}$ of the protein solution and $5 \mu \mathrm{l}$ of the precipitating solution (24 \% PEG 3350, 0.2 M magnesium chloride, 0.1

211 M Hepes, $\mathrm{pH}$ 8.0). After one day, crystals of different morphology, with maximum size of about $2120.05 \mathrm{~mm}$, appeared in the drop.

\section{Data collection and crystallographic analysis}

214 The crystals of the GH9-CBM3c $L$ complex were harvested from the crystallization drop using 215 a nylon cryo-loop (Hampton Research, Aliso Viejo, CA). For data collection, crystals were 216 mounted on the MiTeGen stiff micro-mount (MiTeGen, Ithaca, NY) made of polyimide and

217 flash-cooled in a nitrogen stream produced by Oxford Cryostream low temperature generator

218 (Cosier \& Glazer 1986) at a temperature of $100 \mathrm{~K}$. Mother-liquor of the crystals served for cryo219 protection during the cooling in liquid nitrogen.

220 Diffraction data from the GH9-CBM3c $L$ crystals were measured using the ID23-2 beam line 221 at ESRF, Grenoble, France. A MAR CCD 225 area detector and X-ray radiation of $0.873 \AA$ 
222 wavelength were used. Diffraction data of 480 images with $0.5^{\circ}$ oscillation per image were 223 collected. Data were processed with DENZO and scaled with SCALEPACK as implemented in $224 H K L 2000$ (Otwinowski \& Minor 1997). The crystals diffracted to $1.68 \AA$ resolution and belong 225 to the orthorhombic space group $\mathrm{P} 22_{1} 2_{1} 2_{1}$, with unit cell parameters $\mathrm{a}=70.4, \mathrm{~b}=88.5, \mathrm{c}=106.5 \AA$. 226 There is one GH9-CBM3c $L$ complex per asymmetric unit with a Matthews density $\mathrm{V}_{\mathrm{M}}$ of 2.37 $227 \AA^{3} \mathrm{Da}^{-1}$, corresponding to a solvent content of $48.15 \%$ (Matthews 1968 ). The X-ray data analysis 228 statistics are presented in Table 1 (Stout \& Jensen 1968).

229 Molecular replacement was carried out with MOLREP (Vagin \& Teplyakov 1997), using the 230 coordinates of the GH9 and CBM3c modules of endoglucanase 9G from Clostridium

231 cellulolyticum (PDB code 1G87, 66 and 51\% sequence identity, respectively), as a search model.

232 The MOLREP calculations with the GH9 domain converged into a clear solution with 1 molecule 233 in the asymmetric unit with an R-factor of 0.533 and correlation coefficient of 0.567 . This 234 solution was inserted into MOLREP calculations as a fixed molecule and the coordinates of 235 CBM3c module were used for the search producing a solution with an $\mathrm{R}_{\text {cryst }}$ of 0.505 , and 236 correlation coefficient of 0.582 . The resulting model with $5 \%$ of reflections forming test set

237 (Brünger 1992) was subjected to 10 cycles of restrained refinement using anisotropic B-factors, 238 yielding the $\mathrm{R}_{\text {cryst }}$ and $\mathrm{R}_{\text {free }} 0.329$ and 0.359, respectively (REFMAC5) (Murshudov et al. 1997). 239 Automated model building by ARP/wARP (Perrakis et al. 1999) produced a complete structure 240 with $\mathrm{R}_{\text {cryst }}$ and $\mathrm{R}_{\text {free }}$ of 0.218 and 0.243 respectively. The model was manually corrected using 241 COOT (Emsley \& Cowtan 2004) and refined using REFMAC5 (Murshudov et al. 1997). The $242 \mathrm{R}_{\text {cryst }}$ and $\mathrm{R}_{\text {free }}$ improved to 0.184 and 0.228 , respectively. Solvent atoms were built using $243 A R P /$ warp (Perrakis et al. 1999). Refinement of TLS (rigid body translation/libration/screw 244 motions) parameters was performed (Winn et al. 2001; Winn et al. 2003). The model was 245 subjected to several additional cycles of manual rebuilding and refinement. The model 246 converged to final $\mathrm{R}_{\text {cryst }}$ and $\mathrm{R}_{\text {free }}$ factors of 0.144 and 0.176 , respectively.

247 The refinement statistics of the structure are summarized in Table 2. The structure was 248 validated using MolProbity (Davis et al. 2007).

Protein sequence analysis

250 Sequence alignments were performed using CLUSTALW (Larkin et al. 2007) and the coloring 251 of residues (representing degree of conservation) using ProtSkin (Deprez et al. 2005). Sources of 
252 the sequences used in this work are as follows: Clostridium thermocellum Ce19I GH9 module, 253 CBM3c and CBM3b (AAA20892.1); Clostridium cellulolyticum Cel9G GH9 module, CBM3c 254 (AAA73868.1); Thermobifida fusca Cel9A GH9 module and CBM3c (AAB42155.1); 255 Cellulomonas fimi Ce9A CBM3c (AAA23086.1); Clostridium cellulovorans EngH CBM3c 256 (AAC38572.2) and CbpA CBM3a (AAA23218.1); Clostridium stercorarium CelZ CBM3c and 257 CBM3b (CAA39010.1) and CelY CBM3b (CAA93280.1); Clostridium thermocellum CipA 258 CBM3a (CAA48312.1), CelQ CBM3c (BAB33148.1), Cel9V CBM3c' and CBM3b' 259 (CAK22315.1), Ce19U CBM3c' and CBM3b' (CAK22317.1) and Cbh9A CBM3b 260 (CAA56918.1); Clostridium cellulolyticum CipC CBM3a (AAC28899.2) and CelJ CBM3c 261 (AAG45158.1); Acetivibrio cellulolyticus Ce19B CBM3c' and CBM3b' (CAI94607.1) and CipV 262 (ScaA) CBM3b (AAF06064.1); Clostridium josui CipA (CipJ) CBM3a (BAA32429.1); 263 Bacteroides cellulosolvens ScaA CBM3b (AAG01230.2); Bacillus subtilis CelA CBM3b 264 (AAA22307.1); Pectobacterium atrosepticum CelVI CBM3b (X79241.2); Bacillus 265 licheniformis CelA CBM3b (CAJ70714.1).

\section{Results}

\section{Cloning, expression and purification of Cel9I and its modular components}

270

The full-length $C$. thermocellum Ce19I enzyme and its individual component parts were overexpressed in Escherichia coli, according to Burstein et al (2009), in order to investigate the 272 contribution of the ancillary modules and their linkers to the catalytic activity of the enzyme.

273 These include the isolated GH9 module with and without a His tag, the His-tagged CBM3c 274 module together with its adjacent N-terminal linker that connects it to the GH9 module $275(\mathrm{CBM} 3 \mathrm{c} L)$ and His-tagged $\mathrm{CBM} 3 \mathrm{c}$ module without the $\mathrm{N}$-terminal linker (CBM3cNL). For 276 details, see Figure 1. Following purification procedures, all recombinant proteins showed a 277 single band in SDS-PAGE of the anticipated molecular masses. 
Recovery of endoglucanase activity upon association of CBM3cNL and GH9 compared to CBM3cL and GH9

Previous works (Burstein et al. 2009; Gilad et al. 2003) demonstrated that the Cel9I catalytic module alone has no detectable activity on CMC (carboxymethyl cellulose) and that adding the $\mathrm{CBM} 3 \mathrm{c} L$ to form the Ce19I-CBM3c $L-\mathrm{CBM} 3 \mathrm{~b}$ triad serves to recover up to $70 \%$ of the lost activity. To further examine the importance of the linker connecting the GH9 and the CBM3c modules, we tested the ability of CBM3cNL to recover the CMCase activity of GH9. A fixed amount of the catalytic module $(70 \mathrm{pmol}$ in $400 \mu \mathrm{l})$ was mixed with increasing amounts of $\mathrm{CBM} 3 \mathrm{c} L$ or $\mathrm{CBM} 3 \mathrm{c} N L$. The activity of the intact Ce19I enzyme was defined as $100 \%$, and the activity of the reconstituted complexes was measured relative to that of Cel9I. The results indicated that GH9-CBM3cNL exhibit only about $10 \%$ of the intact Ce19I activity towards CMC, whereas the reassembled GH9-CBM3cL provided up to $50 \%$ of the activity (Figure 2 ). The fact that a higher than one molar ratio was required to obtain maximum activity can be explained by the fact that the CBM protein was only partly functional as was also observed in the ITC experiments described below. Overall the results suggest that the linker is required for better fitting of the reconstituted $\mathrm{CBM} 3 \mathrm{c}$ which results in better recovered activity.

\section{Overall structure of the reassembled GH9-CBM3c}

Initially, we tried to overexpress and purify the covalently linked GH9-CBM3c, however the full-length protein was unstable and proteolysis occurred during the overexpression and purification stages, partially resulting in separate GH9 and CBM3c modules. Therefore the obtained protein samples were not homogenous and were not suitable for crystallization trials. Instead, we employed an alternative approach where we expressed the two domains separately and combined them in vitro. Surprisingly, the combined modules crystallized and formed a structure similar to those of the known GH9 cellulases.

The crystal structure of the reassembled C. thermocellum Ce19I GH9-CBM3c $L$ dyad was 303 determined by molecular replacement and the coordinates are deposited in Protein Data Bank with code 2XFG. Data collection and refinement statistics are given in Tables 1 and 2. The catalytic GH9 and the ancillary CBM3c modules reassembled in vitro to form a dyad (Figure 3a) similar in structure to the intact tandem GH9-CBM3c modules of the orthologous 
307 endoglucanases: Ce19G from C. cellulolyticum (1G87) and Ce19A (previously termed cellulase

308 E4) from Thermobifida fusca (1TF4), with an RMS deviation of $0.783 \AA$ over $468 \mathrm{C} \alpha$ atoms

309 with Cel9G and $0.757 \AA$ with Ce19A (Figure 3b).

\section{Structure of the GH9 module}

311 The catalytic module of the Cel9I enzyme consists of residues 1-446, comprising $15 \alpha$ -

312 helices, whereby the twelve longest ones form the $(\alpha / \alpha)_{6}$-barrel (Figure $\left.4 \mathrm{~A}\right)$. The hydrophobic

313 core of the GH9 module is formed by 118 hydrophobic and aromatic amino acids, the vast

314 majority of which are also conserved in the GH9 modules from C. cellulolyticum Cel9G and $T$.

315 fusca Ce19A. Hydrophobic and aromatic cores have been proposed to play an important role in

316 the formation of $(\alpha / \alpha)_{6}$-barrels (Mandelman et al. 2003). The GH9 module of Ce19I thus shows

317 high structural similarity with the two latter GH9 structures: C. cellulolyticum Cel9G $(0.367 \AA$

318 RMS deviation over 349 C-alpha atoms) and T. fusca Ce19A (0.532 ̊ RMS deviation over 359

319 C-alpha atoms).

320 The catalytic site of the GH9 module is located at the depression in the flat surface, formed by

321 the loops connecting the $\mathrm{N}$ termini of the barrel helices (Figure 4B). The flat face is rich in

322 charged and polar residues (Figure 4B), highly conserved also in Cel9G (1G87) and Cel9A

323 (1TF4). The GH9 modules of these cellulases (Mandelman et al. 2003; Sakon et al. 1997; Zhou

324 et al. 2004) exhibit similar flat faces and clefts, and these conserved residues (His 126, Trp 129,

325 Phe 205, Tyr 206, Trp 209, Trp 256, Asp 261, Asp 262, Trp 314, Arg 318, His 376, Arg 378, and

326 Tyr 419) have been shown to bind natural and synthetic oligosaccharides (Figure 4C). In the

327 present structure, as in the other known GH9-CBM3c bimodular structures, one end of this cleft

328 is blocked by a loop formed by residues 243-254 and the other end is fused with the flat surface

329 of the CBM3c module (Figure 4B). Details of the catalytic cleft are presented in Figure 4C.

330 One calcium ion is found near the catalytic cleft of the GH9 module of Ce19I and is

331 coordinated by a Ser 210 (OG) $2.6 \AA$, Gly 211 (O) $2.4 \AA$, Asp 261 (O) $2.4 \AA$, Asp 214 bifurcated

332 (OD1, OD2) $2.5 \AA$, and Glu 215 bifurcated (OD1, OD2) $2.5 \AA$ (Figure 4D). Despite some minor

333 changes in the residues of coordination this ion seems to be structurally equivalent to those of $T$.

334 fusca Ce19A (RMS deviation $0.160 \AA$ over $5 \mathrm{C} \alpha$ atoms of the coordinating residues), and $C$.

335 cellulolyticum Ce19G (RMS deviation $0.503 \AA$ over $4 \mathrm{C} \alpha$ atoms). In all three cases the calcium

336 ion draws together the $\mathrm{N}$-terminal ends of $\alpha$-helixes 8 and 10 . 


\section{Structure of the CBM $3 \mathrm{c}$ module}

338 The CBM3c module consists of 150 amino acids arranged in an eight $\beta$-stranded sandwich 339 motif homologous to other known family 3 CBM structures (Gilbert et al. 2013; Mandelman et 340 al. 2003; Petkun et al. 2010b; Sakon et al. 1997; Shimon et al. 2000b; Tormo et al. 1996; Yaniv 341 et al. 2014; Yaniv et al. 2012b; Yaniv et al. 2011). The "lower" face of the sandwich is formed

342 by $\beta$-strands 1, 2, and 7; the "upper" face is formed by $\beta$-strands 3, 3', 6, 8, and 9 (Figure 5A).

343 The structure of Ce19I CBM3c is particularly similar to the structures of the other two previously

344 described CBM3c structures (RMS deviation $0.734 \AA$ over $116 \mathrm{C}$-alpha atoms with CBM3c from

345 C. cellulolyticum Ce19G; RMS deviation 0.829 Å over 113 atoms with CBM3c from T. fusca

346 Ce19A). Only $31 \%$ of amino acids are located in $\beta$-strands of the CBM3c module from Cel9I;

347 others are found in the loop regions.

348 One calcium ion was found in the upper $\beta$-sheet of the CBM3c molecule (Figure 5B) and is

349 coordinated by a water molecule and five residues from the upper $\beta$-sheet: Asn $500(\mathrm{O})$, Glu 503

350 bifurcated (OE1, OE2), Asn 573 (O), Asn 576 (OD1), Asp 577 (OD1). This calcium atom is in a

351 similar location as in Ce19A and Ce19G, and probably plays a structural role for most CBM3

352 modules, as was suggested previously (Tormo et al. 1996).

353 The lower sheet forms a flat platform conserved between the CBM3c modules and the other

354 two molecular structures. This flat surface is rich in charged and polar conserved surface

355 residues: Asn 466, Glu 474, Lys 476, Ser 518, Tyr 520, Glu 559, Gln 561, and Arg 563 (Figure

356 5C). The planar region of the CBM3c modules in all three enzymes is particularly aligned in

357 continuation of the catalytic cleft of the catalytic modules, and has been proposed to bind single

358 chains of cellulose and guide them to the cleft (Mandelman et al. 2003; Sakon et al. 1997).

359 The CBM3c possesses a very interesting surface structure, formed by the $\beta$-strands on the

360 opposite side of the flat surface, called the "shallow groove" (Shimon et al. 2000b; Tormo et al.

361 1996). The "shallow groove" is lined by four aromatic rings (Phe 498, Tyr 538, Tyr 578 and Tyr

362 597), two charged or polar residues (Arg 496, and Glu 540), Leu 602, Pro 595 and Pro 608.

363 These residues are also conserved in other CBM3 modules regardless of their subgroup relation

364 (a, b, or c), their cellulose-binding ability and their effect on the activity of the catalytic module.

365 Figure 5D shows the shallow groove of the CBM3c module from the Cel9I enzyme colored

366 according to the extent of the conservation of the residues in other CBM3a, b and c modules 
367 (darker blue represents more conservation). The alignment was performed over 25 CBM3 368 sequences (11 CBM3c, 12 CBM3b and CBM3b', and 4 CBM3a). Conservation of this surface

369 structure, regardless of the particular known function of the CBMs, implies that this site has

370 some kind of "generic" function. This shallow groove may serve to bind to single

371 oligosaccharide chains or to peptide chains, such as the intermodular linkers common to

372 cellulases or cellulosomal scaffoldin subunits. There is evidence that the shallow groove interacts

373 with a linker region (Petkun et al. 2010a; Shimon et al. 2000a; Yaniv et al. 2012a).

374 Contact residues between the GH9, linker and CBM3c

375 The in vitro reassembled GH9-CBM3c $L$ complex has a large intermodular interface, the 376 contact area of which is $1108.3 \AA^{2}$, corresponding to $12.3 \%$ of the total surface-exposed area of 377 the CBM3c module and $6.2 \%$ of the exposed GH9 module (Krissinel \& Henrick 2007). The GH9 378 and the $\mathrm{CBM} 3 \mathrm{c} L$ modules of Ce19I are assembled into the reconstituted GH9-CBM3c complex 379 by 31 hydrogen bonds ( 4 main chain-main chain, 19 main chain-side chain, and 8 side chain-side 380 chain), 14 hydrophobic, 3 aromatic interactions, and 3 ionic bonds

381 (http://pic.mbu.iisc.ernet.in/index.html) (Tina et al. 2007). Sixteen residues from the GH9

382 module and seventeen residues from the CBM3c participate in these interactions (contact

383 residues are shown in Figure 6A). The vast majority of the contact residues and contacts are

384 similar to those of C. cellulolyticum Ce19G and of T. fusca Ce19A (Figure 6B).

385 Conserved residues of the linker (which spans from Gly447 to Asp462) make numerous 386 contacts with conserved residues of the GH9 module, emphasizing the importance of the linker 387 in this interaction (Figure 6). A conserved Gly447 of the linker interacts via hydrogen bonds 388 with Gly444 and Tyr440 of the GH9 module. Pro449 forms hydrophobic interactions with 389 Tyr440, and Asp450 forms hydrogen bonds with Gln11. Another linker residue, Phe453, forms 390 hydrophobic and aromatic interactions with two aromatic residues of the GH9 module, i.e., 391 Phe15 and Tyr399. Gly455 forms hydrogen bonds with Asn33 and Arg31. Glu457 forms 392 intricate interactions with a variety of residues of the GH9 module, which include hydrogen 393 bonding with Asn33, Val397, Arg395, as well as hydrogen and ionic interactions with His396.

394 Glu461 exhibits hydrogen and ionic interactions with Arg395. Additionally, linker residues

395 Glu457 and Glu461 form hydrogen bonds and salt bridges with Trp490 and Lys487, 396 respectively. The latter belong to the CBM3c module and are part of a loop (486-498), which 
397 protrudes towards and forms interactions with several amino acids of the GH9 module. There are

398 also many hydrogen bonds formed between the neighboring amino acids of the linker, thus

399 contributing to its defined conformation. Altogether the multiple, well-conserved interactions

400 between the linker, the GH9 and the CBM3c modules stabilize the spatial orientation of the

401 modules towards one another and contribute to the structural rigidity of the entire molecule,

402 resulting in an active enzyme structure.

403 As mentioned above, the mutual spatial orientation of the GH9 and CBM3c modules is very 404 similar to that in the native, intact bimodular pairs from Cel9G and Cel9A leading to the overall

405 similarity in structures. The remarkable conservation of the overall architecture in the

406 reassembled in vitro complex together with the striking conservation of the contact residues

407 implies its high functional importance. In all of these structures (Ce19G, Ce19A, and the

408 reassembled GH9-CBM3c $L$ from Ce19I), the flat surface of the CBM3c module is aligned in

409 continuation with the catalytic cleft of the GH9 module, making an extended platform (Figure

410 4B). This platform is rich in charged and polar surface residues that are highly conserved

411 throughout the family 3 CBMc's.

\section{Microcalorimetric analysis of the GH9-CBM3c complex formation}

413 The binding constants of GH9 and the CBM3c were obtained by performing isothermal

414 titration calorimetry (ITC) experiments in which a solution of GH9 was titrated with a solution

415 of CBM3c with or without the linker (Figure 7). Control experiments for each of the

416 components alone were conducted and subtracted from the titration data. In both cases the

417 titration curve could be fitted to a one-site binding model although the calculated stoichiometry

418 was less than one. The low stoichiometry is probably a result of the fact that the soluble CBM

419 module lost its functionality with time and its true active concentration was less than the

420 measured protein concentration. To estimate the binding constants for the two CBM3c forms the

421 CBM3c concentrations were corrected to provide a stoichiometry of one. In all cases the binding

422 reactions were enthalpy driven with a negative entropy contribution. CBM3cL provided binding

423 constants $\left(\mathrm{K}_{\mathrm{d}}\right)$ between 1.3-2.0 × 10-6 $\mathrm{M}$, whereas CBM3cNL exhibited stronger binding

424 constants, $\mathrm{K}_{\mathrm{d}}$ between 2.9-4.3 $\times 10^{-7} \mathrm{M}$. Thus, the linker may serve as a mitigating factor for the

425 binding process, ensuring specific binding orientation. This is consistent with the structural data

426 and the activity assays, which emphasizes the important role of the linker in enzyme functioning. 
427 In the case of CBM3cNL, the binding process may occur faster in the absence of linker, but may 428 also lead to unspecific binding and aggregation of the modules.

\section{Discussion}

A striking feature of the family 9 glycoside hydrolases is their subdivision into architectural themes, which are defined by their conserved modular composition (Bayer et al. 2006). In this context, the Theme B1 endoglucanases contain a GH9 catalytic module followed by a purportedly fused family $3 \mathrm{c}$ CBM. Biochemical studies of some of the members of this group (Arai et al. 2001; Chiriac et al. 2010; Gal et al. 1997; Irwin et al. 1998; Li et al. 2007) have shown that the CBM3c acts as a modulator of the function of the catalytic module. However, the exact manner in which the CBM3c functions is still unclear. It has been shown (Gal et al. 1997; Gilad et al. 2003; Irwin et al. 1998) that family 3c CBMs (including the CBM3c from $C$. thermocellum Ce19I) fail to bind insoluble cellulosic substrates, implying that they do not act as targeting agents for such substrates. The targeting of the enzyme to crystalline cellulose is achieved either through an additional CBM (Kostylev et al. 2012) or by attachment of the enzyme to a CBM-containing scaffoldin via a cohesin-dockerin interaction (Mingardon et al. 444 2011).

The CBM3c module of Cel9A from the T. fusca has been proposed to loosely anchor the enzyme to cellulose, to disrupt the hydrogen bonds in crystalline cellulose and to guide a single cellulose strand towards the active site of the GH9 catalytic module (Bayer et al. 2006; Li et al. 2007). This hypothesis has been supported by molecular docking and molecular dynamics simulation studies (Oliveira et al. 2009). Moreover, double point mutations indicated that high coordination between the substrate affinities of the catalytic module and CBM needs to be precisely controlled ( $\mathrm{Li}$ et al. 2010). Enzyme thermostability was reported to be affected by the presence of the $\mathrm{CBM} 3 \mathrm{c}$ probably due to the formation of a compact structure (Chiriac et al. 454 2010; Su et al. 2012; Yi et al. 2013). 
The previously reported structures of Cel9A from T. fusca (Sakon et al. 1997) and Cel9G

457

458

459

460

461

462

463

464

465

466

467

468

469

470

471

472

473

474

475

476

477

478

479

480

481

482

483

484

485

486

from C. cellulolyticum (Mandelman et al. 2003) revealed that the catalytic module and the

CBM3c are separated by a $\sim 20$-residue linker that forms multiple polar and hydrophobic

interactions mainly with the GH9 module. In an earlier report, we demonstrated that separately expressed GH9 and $\mathrm{CBM} 3 \mathrm{c} L$ from Ce19I of $C$. thermocellum interact with one another to form an enzymatically active complex (Burstein et al. 2009). In the current article, we showed further that the GH9 and CBM3c can also be reassembled without the linker, albeit at the expense of catalytic activity, thus emphasizing the importance of the linker in positioning correctly the CBM relative to the GH9 catalytic module.

There is evidence that linkers in multi-modular proteins may serve communication roles between the modules via allosteric mechanisms and variation in their sequences affect enzyme activity (Ma et al. 2011). Linker length and rigidity was shown to play a critical role in the cooperative action of the catalytic module of a cellulase and a CBM (Ting et al. 2009).

Computational studies of $T$. fusca Ce19A suggested that thermal contributions to enzyme plasticity and molecular motion at high temperatures may play a role in enhancing CBM and catalytic domain synergy, and the linker may have an important role in this process (Batista et al. 2011). The length of the linkers may also play an important role in protein function and adaptation to the environment (Sonan et al. 2007). Studies in cellulolytic fungi revealed that linkers undergo modifications such as glycoslation and have also been shown to directly bind to the cellulose substrate (Beckham et al. 2012; Payne et al. 2013; Sammond et al. 2012; Srisodsuk et al. 1993). Point mutations in different fungal GH-CBM linkers have also been shown to significantly affect the activity of the enzymes and their stability (Couturier et al. 2013; Lu et al. 2014).

The characteristics of the reassembled linker-containing complex are corroborated by the $\mathrm{X}$-ray crystallographic data. Indeed, it is quite surprising that the two separately expressed entities recombined in such a way that the complex could in fact be crystallized. Moreover, the resultant structure was remarkably similar to the known structures of the intact bimodular GH9CBM3c pairs from C. cellulolyticum Cel9G and T. fusca Cel9A. Accordingly, the vast majority of the contact residues are similar among the three structures. Conserved residues of the linker 
487 make contacts with conserved residues of the GH9 module, highlighting the importance of the 488 linker in this interaction. Multiple hydrogen, ionic and hydrophobic bonds between the linker 489 and the functional GH9 and CBM3c stabilize the spatial organization of the modules. The 490 similarity of the reassembled and native intact structures is particularly intriguing, as it suggests 491 that folding of the modular structures and emplacement of the linker during biosynthesis and 492 intermodular recognition during complex formation are governed by the same interactions, 493 which may have distinct functional consequences. In contrast to the GH9-CBM3cL, the re494 associated GH9-CBM3cNL complex never crystallized, suggesting that the reassembly of the 495 two modules in the absence of linker was somewhat heterogeneous in character.

Single proteins commonly fold into defined structures, wherein their N- and C-terminal ends are in relatively close proximity to one another. If we view the structures of the Theme B1 enzymes, it is evident that their individual modules, the GH9 catalytic module and the CBM3c, are consistent with this rule. The positions of the $\mathrm{N}$ - and $\mathrm{C}$-termini of the Theme B1 catalytic module are similar to those of the other GH9 thematic members, including those of Theme A, which lack additional modules. Likewise, the $\mathrm{N}$ - and $\mathrm{C}$-termini of CBM3c are essentially the same as all other members of the family $3 \mathrm{CBMs}$, regardless of their source (i.e., parent cellulase, scaffoldin, etc). The evolutionary significance of this observation is that, originally, the functional relationship between the two modules was likely a more conventional one, whereby an ancestral CBM3 played a standard targeting role to deliver the GH9 catalytic module to its substrate. During the course of evolution, this relationship changed, and the precise positioning and fusion of a mutated CBM3 with a GH9 catalytic module served to modulate the activity characteristics of the latter. For this purpose, the flat surface of the CBM3c is aligned with the flat surface of the catalytic module, and the appropriate residues that interact with the single cellulose chain are thus aligned with the active site of the GH9 module. As a consequence, the 513 positioning and fusion of the two modules, however, are at odds with the inherent locations of 514 the termini of the two modules, such that the C-terminus of the catalytic module is very distant 515 from the N-terminus of the CBM3c. Consequently, nature has provided a very distinctive type of 516 conserved linker, which both connects the two modules and helps secure their required 517 orientation for processive endoglucanase activity. 


\section{Conclusions}

520 Cellulase 9I (Ce19I), a non-cellulosomal family 9 processive endoglucanase from Clostridium thermocellum, which degrades crystalline cellulose phosphoric acid-swollen cellulose (PASC)

522 and carboxymethyl cellulose (CMC), consists of a catalytic GH9 module followed by two family

5233 carbohydrate-binding modules (CBMs): CBM3c and CBM3b, separated by linker regions. C-

524 terminal CBM3b module, as a classic CBM3, is responsible for targeting the Ce19I enzyme to the

525 planar surface of the crystalline cellulose. The CBM3c is crucial for the GH9 enzymatic activity.

526 In this work we investigated the interaction of separately expressed catalytic module and CBM3c

527 either with or without the intermodular linker in order to better understand the function of the

$528 \mathrm{CBM} 3 \mathrm{c}$ in the family-9 enzymes and the role of the linkers regions.

529 GH9 catalytic module and CBM3c were able to interact and reassemble both with and without

530 the linker; however the linker was essential for the endoglucanase catalytic activity.

531 Surprisingly, we were able to crystallize these two separately expressed entities, meaning that

532 their reassembly was very ordered and structurally homogeneous. The molecular structure of the

533 GH9 and CBM3c with the linker region showed that they form a complex similar in structure to

534 the intact tandem GH9-CBM3c modules of the orthologous endoglucanases Cel9G from $C$.

535 cellulolyticum and Ce19A from Thermobifida fusca. The flat, conserved surface of the CBM3c

536 module is aligned in continuation with the catalytic cleft of the GH9 module, presumably

537 forming one functional entity, which binds to the planar surface of the cellulose. Conserved

538 residues of the linker make contacts with conserved residues of the GH9 module, highlighting

539 the importance of the linker in this interaction. Overall our results demonstrate that the linker

540 regions in the $\mathrm{GH} 9 / \mathrm{CBM} 3 \mathrm{c}$ endoglucanases are necessary to achieve the right spatial

541 organization of the modules and for the fixation of the modules into functional enzymes.

542

\section{Acknowledgements}

544 This article is dedicated to the memory of Professor Felix Frolow, who passed away on 29

545 August 2014. We thankfully acknowledge the ESRF for synchrotron beam time and staff 546 scientists of the ID-29 beam line for their assistance. 
548

549

550

551

552

553

554

555

556

557

558

559

560

561

562

563

564

565

566

567

568

569

570

571

572

573

574

575

576

577

578

579

580

581

582

583

584

585

586

587

588

589

590

591

\section{References}

Akinosho H, Yee K, Close D, and Ragauskas A. 2014. The emergence of Clostridium thermocellum as a high utility candidate for consolidated bioprocessing applications. Front Chem 2:66.

Arai T, Ohara H, Karita S, Kimura T, Sakka K, and Ohmiya K. 2001. Sequence of celQ and properties of celQ, a component of the Clostridium thermocellum cellulosome. Appl Microbiol Biotechnol 57:660-666.

Batista PR, de Souza Costa MG, Pascutti PG, Bisch PM, and de Souza W. 2011. High temperatures enhance cooperative motions between CBM and catalytic domains of a thermostable cellulase: mechanism insights from essential dynamics. Phys Chem Chem Phys 13:13709-13720.

Bayer EA, Belaich J-P, Shoham Y, and Lamed R. 2004. The cellulosomes: Multi-enzyme machines for degradation of plant cell wall polysaccharides. Annu Rev Microbiol 58:521554.

Bayer EA, Lamed R, White BA, and Flint HJ. 2008. From cellulosomes to cellulosomics. Chem Rec 8:364-377.

Bayer EA, Morag E, Lamed R, Yaron S, and Shoham Y. 1998. Cellulosome structure: fourpronged attack using biochemistry, molecular biology, crystallography and bioinformatics. In: Claeyssens M, Nerinckx W, and Piens K, eds. Carbohydrases from Trichoderma reesei and other microorganisms. London: The Royal Society of Chemistry, 39-65.

Bayer EA, Shoham Y, and Lamed R. 2006. Cellulose-decomposing prokaryotes and their enzyme systems. In: Dworkin M, Falkow S, Rosenberg E, Schleifer K-H, and Stackebrandt E, eds. The Prokaryotes, Third Edition. New York: Springer-Verlag, 578617.

Bayer EA, Smith SP, Noach I, Alber O, Adams JJ, Lamed R, Shimon LJW, and Frolow F. 2009. Can we crystallize a cellulosome? In: Sakka K, Karita S, Kimura T, Sakka M, Matsui H, Miyake H, and Tanaka A, eds. Biotechnology of lignocellulose degradation and biomass utilization: Ito Print Publishing Division, 183-205.

Beckham GT, Dai Z, Matthews JF, Momany M, Payne CM, Adney WS, Baker SE, and Himmel ME. 2012. Harnessing glycosylation to improve cellulase activity. Curr Opinion Biotechnol 23:338-345.

Beckham GT, Stahlberg J, Knott BC, Himmel ME, Crowley MF, Sandgren M, Sorlie M, and Payne CM. 2014. Towards a molecular-level theory of carbohydrate processivity in glycoside hydrolases. Curr Opinion Biotechnol 27:96-106.

Béguin P, and Alzari PM. 1998. The cellulosome of Clostridium thermocellum. Biochem Soc Trans 26:178-185.

Béguin P, Millet J, and Aubert J-P. 1992. Cellulose degradation by Clostridium thermocellum: From manure to molecular biology. FEMS Microbiol Lett 100:523-528.

Brünger TA. 1992. Free R value: a novel statistical quantity for assessing the accuracy of crystal structures. Nature 355:472-475.

Bu L, Nimlos MR, Shirts MR, Stahlberg J, Himmel ME, Crowley MF, and Beckham GT. 2012. Product binding varies dramatically between processive and nonprocessive cellulase enzymes. J Biol Chem 287:24807-24813. 
592

593

594

595

596

597

598

599

600

601

602

603

604

605

606

607

608

609

610

611

612

613

614

615

616

617

618

619

620

621

622

623

624

625

626

627

628

629

630

631

632

633

634

635

636

637

Burstein T, Shulman M, Jindou S, Petkun S, Frolow F, Shoham Y, Bayer EA, and Lamed R. 2009. Physical association of the catalytic and helper modules of a processive family-9 glycoside hydrolase is essential for activity. FEBS Lett 583:879-884.

Cantarel BL, Coutinho PM, Rancurel C, Bernard T, Lombard V, and Henrissat B. 2009. The Carbohydrate-Active Enzymes database (CAZy): an expert resource for glycogenomics. Nucl Acids Res 37:D233-238.

Chayen NE, Shaw Stewart PD, Maeder DL, and Blow DM. 1990. An automated system for micro-batch protein crystallization and screening. J Appl Crystallogr 23:297-302.

Chiriac AI, Cadena EM, Vidal T, Torres AL, Diaz P, and Pastor FI. 2010. Engineering a family 9 processive endoglucanase from Paenibacillus barcinonensis displaying a novel architecture. Appl Microbiol Biotechnol 86:1125-1134.

Cosier J, and Glazer AM. 1986. A nitrogen-gas-stream cryostat for general X-ray-diffraction studies. J Appl Crystalogr 19:105-107.

Couturier M, Feliu J, Bozonnet S, Roussel A, and Berrin JG. 2013. Molecular engineering of fungal GH5 and GH26 beta-(1,4)-mannanases toward improvement of enzyme activity. PloS ONE 8:e79800.

Cuskin F, Flint JE, Gloster TM, Morland C, Basle A, Henrissat B, Coutinho PM, Strazzulli A, Solovyova AS, Davies GJ, and Gilbert HJ. 2012. How nature can exploit nonspecific catalytic and carbohydrate binding modules to create enzymatic specificity. Proc Natl Acad Sci U S A 109:20889-20894.

Davies G, and Henrissat B. 1995. Structures and mechanisms of glycosyl hydrolases. Structure 3:853-859.

Davis IW, Leaver-Fay A, Chen VB, Block JN, Kapral GJ, Wang X, Murray LW, Arendall WB, 3rd, Snoeyink J, Richardson JS, and Richardson DC. 2007. MolProbity: all-atom contacts and structure validation for proteins and nucleic acids Nucl Acids Res 35(Web Server issue):W375-383.

Demain AL, Newcomb M, and Wu JH. 2005. Cellulase, clostridia, and ethanol. Microbiol Mol Biol Rev 69:124-154.

Deprez C, Lloubes R, Gavioli M, Marion D, Guerlesquin F, and Blanchard L. 2005. Solution structure of the E. coli TolA C-terminal domain reveals conformational changes upon binding to the phage g3p N-terminal domain J Mol Biol 346:1047-1057.

Doi RH, and Kosugi A. 2004. Cellulosomes: plant-cell-wall-degrading enzyme complexes. Nat Rev Microbiol 2:541-551.

Emsley P, and Cowtan K. 2004. Coot: model-building tools for molecular graphics. Acta Crystallogr D 60:2126-2132.

Felix CR, and Ljungdahl LG. 1993. The cellulosome - the exocellular organelle of Clostridium. Annu Rev Microbiol 47:791-819.

Fontes CM, and Gilbert HJ. 2010. Cellulosomes: Highly efficient nanomachines designed to deconstruct plant cell wall complex carbohydrates. Annu Rev Biochem 79:655-681.

Freier D, Mothershed CP, and Wiegel J. 1988. Characterization of Clostridium thermocellum JW20. Appl Environ Microbiol 54:204-211.

Gal L, Gaudin C, Belaich A, Pagès S, Tardif C, and Belaich J-P. 1997. CelG from Clostridium cellulolyticum: a multidomain endoglucanase acting efficiently on crystalline cellulose. $J$ Bacteriol 179:6595-6601.

Garcia-Martinez DV, Shinmyo A, Madia A, and Demain AL. 1980. Studies on cellulase production by Clostridium thermocellum. Eur J Appl Microbiol Biotechnol 9:189-197.

Peer] reviewing PDF | (2015:05:5166:1:0:NEW 29 Jun 2015) 
638

639

640

641

642

643

644

645

646

647

648

649

650

651

652

653

654

655

656

657

658

659

660

661

662

663

664

665

666

667

668

669

670

671

672

673

674

675

676

677

678

679

680

681

Gilad R, Rabinovich L, Yaron S, Bayer EA, Lamed R, Gilbert HJ, and Shoham Y. 2003. CelI, a non-cellulosomal family-9 enzyme from Clostridium thermocellum, is a processive endoglucanase that degrades crystalline cellulose. J Bacteriol 185:391-398.

Gilbert HJ, and Hazlewood GP. 1993. Bacterial cellulases and xylanases. J Gen Microbiol 139:187-194.

Gilbert HJ, Knox JP, and Boraston AB. 2013. Advances in understanding the molecular basis of plant cell wall polysaccharide recognition by carbohydrate-binding modules. Curr Opin Struct Biol 23:669-677.

Gilkes NR, Henrissat B, Kilburn DG, Miller RCJ, and Warren RAJ. 1991. Domains in microbial b-1,4-glycanases: sequence conservation, function, and enzyme families. Microbiol Rev 55:303-315.

Gruninger RJ, Gong X, Forster RJ, and McAllister TA. 2014. Biochemical and kinetic characterization of the multifunctional beta-glucosidase/beta-xylosidase/alphaarabinosidase, Bgxa1. Appl Microbiol Biot 98:3003-3012.

Henrissat B. 1991. A classification of glycosyl hydrolases based on amino acid sequence similarities. Biochem J 280:309-316.

Henrissat B, and Davies G. 1997. Structural and sequence-based classification of glycoside hydrolases. Curr Opin Struct Biol 7:637-644.

Henrissat B, and Davies GJ. 2000. Glycoside hydrolases and glycosyltransferases. Families, modules, and implications for genomics. Plant Physiol 124:1515-1519.

Henrissat B, and Romeu A. 1995. Families, superfamilies and subfamilies of glycosyl hydrolases. Biochem J 311:350-351.

Irwin D, Shin D-H, Zhang S, Barr BK, Sakon J, Karplus PA, and Wilson DB. 1998. Roles of the catalytic domain and two cellulose binding domains of Thermomonospora fusca E4 in cellulose hydrolysis. J Bacteriol 180:1709-1714.

Jeon SD, Yu KO, Kim SW, and Han SO. 2012. The processive endoglucanase EngZ is active in crystalline cellulose degradation as a cellulosomal subunit of Clostridium cellulovorans. N Biotechnol 29:365-371.

Kostylev M, Moran-Mirabal JM, Walker LP, and Wilson DB. 2012. Determination of the molecular states of the processive endocellulase Thermobifida fusca Ce19A during crystalline cellulose depolymerization. Biotechnol Bioeng 109:295-299.

Krissinel E, and Henrick K. 2007. Inference of macromolecular assemblies from crystalline state. J Mol Biol 372:774--797.

Kuusk S, Sorlie M, and Valjamae P. 2015. The predominant molecular state of bound enzyme determines the strength and type of product inhibition in the hydrolysis of recalcitrant polysaccharides by processive enzymes. J Biol Chem. 290: 11678-11691

Lamed R, Setter E, and Bayer EA. 1983a. Characterization of a cellulose-binding, cellulasecontaining complex in Clostridium thermocellum. J Bacteriol 156:828-836.

Lamed R, Setter E, Kenig R, and Bayer EA. 1983b. The cellulosome - a discrete cell surface organelle of Clostridium thermocellum which exhibits separate antigenic, cellulosebinding and various cellulolytic activities. Biotechnol Bioeng Symp 13:163-181.

Larkin MA, Blackshields G, Brown NP, Chenna R, McGettigan PA, McWilliam H, Valentin F, Wallace IM, Wilm A, Lopez R, Thompson JD, Gibson TJ, and Higgins DG. 2007. ClustalW and ClustalX version 2. Bioinformatics 23:2947-2948. 
682

683

684

685

686

687

688

689

690

691

692

693

694

695

696

697

698

699

700

701

702

703

704

705

706

707

708

709

710

711

712

713

714

715

716

717

718

719

720

721

722

723

724

725

726

Li Y, Irwin DC, and Wilson DB. 2007. Processivity, substrate binding, and mechanism of cellulose hydrolysis by Thermobifida fusca Ce19A. Appl Environ Microbiol 73:31653172.

Li Y, Irwin DC, and Wilson DB. 2010. Increased crystalline cellulose activity via combinations of amino acid changes in the family 9 catalytic domain and family $3 \mathrm{c}$ cellulose binding module of Thermobifida fusca Ce19A. Appl Environ Microbiol 76:2582-2588.

Lu H, Luo H, Shi P, Huang H, Meng K, Yang P, and Yao B. 2014. A novel thermophilic endobeta-1,4-mannanase from Aspergillus nidulans XZ3: functional roles of carbohydratebinding module and Thr/Ser-rich linker region. Appl Microbiol Biotechnol 98:2155-2163.

Ma B, Tsai CJ, Haliloglu T, and Nussinov R. 2011. Dynamic allostery: linkers are not merely flexible. Structure (London, England : 1993) 19:907-917.

Mandelman D, Belaich A, Belaich JP, Aghajari N, Driguez H, and Haser R. 2003. X-Ray crystal structure of the multidomain endoglucanase Cel9G from Clostridium cellulolyticum complexed with natural and synthetic cello-oligosaccharides. J Bacteriol 185:4127-4135.

Matthews BW. 1968. Solvent content of protein crystals. J Mol Biol 33:491-497.

Meekins DA, Raththagala M, Husodo S, White CJ, Guo HF, Kotting O, Vander Kooi CW, and Gentry MS. 2014. Phosphoglucan-bound structure of starch phosphatase Starch Excess4 reveals the mechanism for C6 specificity. Proc Natl Acad Sci U S A 111:7272-7277.

Miller GL. 1959. Use of dinitrosalicylic acid reagent for determination of reducing sugar. Anal Biochem 31:426-428.

Mingardon F, Bagert JD, Maisonnier C, Trudeau DL, and Arnold FH. 2011. Comparison of family 9 cellulases from mesophilic and thermophilic bacteria. Appl Environ Microbiol 77:1436-1442.

Moraïs S, Alber O, Barak Y, Hadar Y, Wilson DB, Lamed R, Shoham Y, and Bayer EA. 2012. Functional association of the catalytic and ancillary modules dictates enzymatic activity in glycoside hydrolase family $43 \beta$-xylosidase. J Biol Chem 287:9213-9221.

Murshudov GN, Vagin AA, and Dodson EJ. 1997. Refinement of macromolecular structures by the maximum-likelihood method. Acta Crystallogr D 53:240-255.

$\mathrm{Ng} \mathrm{TK}$, Weimer TK, and Zeikus JG. 1977. Cellulolytic and physiological properties of Clostridium thermocellum. Arch Microbiol 114:1-7.

Noach I, Alber O, Bayer EA, Lamed R, Levy-Assaraf M, Shimon LJW, and Frolow F. 2008. Crystallization and preliminary X-ray analysis of Acetivibrio cellulolyticus cellulosomal type II cohesin module: Two versions having different linker lengths. Acta Crystallogr F 64:58-61.

Oliveira OV, Freitas LCG, Straatsma TP, and Lins RD. 2009. Interaction between the CBM of Ce19A from Thermobifida fusca and cellulose fibers. J Mol Recognit 22:38-45.

Otwinowski Z, and Minor W. 1997. Processing of X-ray diffraction data collected in oscillation mode. Meth Enzymol, 307-326.

Payne CM, Bomble YJ, Taylor CB, McCabe C, Himmel ME, Crowley MF, and Beckham GT. 2011. Multiple functions of aromatic-carbohydrate interactions in a processive cellulase examined with molecular simulation. J Biol Chem 286:41028-41035.

Payne CM, Resch MG, Chen L, Crowley MF, Himmel ME, Taylor LE, 2nd, Sandgren M, Stahlberg J, Stals I, Tan Z, and Beckham GT. 2013. Glycosylated linkers in multimodular lignocellulose-degrading enzymes dynamically bind to cellulose. Proc Natl Acad Sci U S A 110:14646-14651. 
727

734

735

736

737

738

739

740

741

742

743

744

745

746

747

748

749

750

751

752

753

754

755

756

757

758

759

760

761

762

763

764

765

766

767

768

769

770

771

772
Perrakis A, Morris R, and Lamzin VS. 1999. Automated protein model building combined with iterative structure refinement. Nat Struct Biol 6:458-463.

Petkun S, Jindou S, Shimon LJW, Rosenheck S, Bayer EA, Lamed R, and Frolow F. 2010a. Structure of a family $3 \mathrm{~b}$ ' carbohydrate-binding module from the Cel9V glycoside hydrolase from Clostridium thermocellum: structural diversity and implications for carbohydrate binding. Acta Crystallogr D 66:33-43.

Petkun S, Jindou S, Shimon LJW, Rosenheck S, Bayer EA, Lamed R, and Frolow F. $2010 \mathrm{~b}$. Structure of a family $3 b^{\prime}$ carbohydrate-binding module from the Cel9V glycoside hydrolase from Clostridium thermocellum. Structural diversity and implications for carbohydrate binding. Acta Cryst D66:33-43.

Sakon J, Irwin D, Wilson DB, and Karplus PA. 1997. Structure and mechanism of endo/exocellulase E4 from Thermomonospora fusca. Nat Struct Biol 4:810-818.

Sammond DW, Payne CM, Brunecky R, Himmel ME, Crowley MF, and Beckham GT. 2012. Cellulase linkers are optimized based on domain type and function: insights from sequence analysis, biophysical measurements, and molecular simulation. PloS one 7:e48615.

Schwarz WH. 2001. The cellulosome and cellulose degradation by anaerobic bacteria. Appl Microbiol Biotechnol 56:634-649.

Schwarz WH, Zverlov VV, and Bahl H. 2004. Extracellular glycosyl hydrolases from Clostridia. Advan Appl Microbiol 56:215-261.

Shimon LJ, Pages S, Belaich A, Belaich JP, Bayer EA, Lamed R, Shoham Y, and Frolow F. 2000a. Structure of a family IIIa scaffoldin CBD from the cellulosome of Clostridium cellulolyticum at 2.2 A resolution. Acta Crystallogr D 56:1560-1568.

Shimon LJW, Pagès S, Belaich A, Belaich JP, Bayer EA, Lamed R, Shoham Y, and Frolow F. 2000b. Structure of a family IIIa scaffoldin CBD from the cellulosome of Clostridium cellulolyticum at $2.2 \AA$ resolution. Acta Crystallogr D 56:1560-1568.

Shoham Y, Lamed R, and Bayer EA. 1999. The cellulosome concept as an efficient microbial strategy for the degradation of insoluble polysaccharides. Trends Microbiol 7:275-281.

Sonan GK, Receveur-Brechot V, Duez C, Aghajari N, Czjzek M, Haser R, and Gerday C. 2007. The linker region plays a key role in the adaptation to cold of the cellulase from an Antarctic bacterium. Bioch J 407:293-302.

Srisodsuk M, Reinikainen T, Penttila M, and Teeri TT. 1993. Role of the interdomain linker peptide of Trichoderma reesei cellobiohydrolase I in its interaction with crystalline cellulose. Journal Biol Chem 268:20756-20761.

Stout GH, and Jensen LH. 1968. X-ray structure determination. A practical guide. London: MacMillan.

Su X, Mackie RI, and Cann IK. 2012. Biochemical and mutational analyses of a multidomain cellulase/mannanase from Caldicellulosiruptor bescii. Appl Environ Microb 78:22302240.

Telke AA, Ghatge SS, Kang SH, Thangapandian S, Lee KW, Shin HD, Um Y, and Kim SW. 2012. Construction and characterization of chimeric cellulases with enhanced catalytic activity towards insoluble cellulosic substrates. Bioresource Technol 112:10-17.

Tina KG, Bhadra R, and Srinivasan N. 2007. PIC: Protein interactions calculator. Nucl Acids Res 35:W473-W476.

Ting CL, Makarov DE, and Wang ZG. 2009. A kinetic model for the enzymatic action of cellulase. J Phys Chem B 113:4970-4977. 
773

774

775

776

777

778

779

780

781

782

783

784

785

786

787

788

789

790

791

792

793

794

795

796

797

798

799

800

801

802

803

804

805

806

807

808

809

810

811

812

813

814

815

816

817

818

Tormo J, Lamed R, Chirino AJ, Morag E, Bayer EA, Shoham Y, and Steitz TA. 1996. Crystal structure of a bacterial family-III cellulose-binding domain: A general mechanism for attachment to cellulose. EMBO J 15:5739-5751.

Vagin A, and Teplyakov A. 1997. MOLREP: an automated program for molecular replacement. $J$ Appl Crystalogr 30:1022-1025.

Venditto I, Najmudin S, Luis AS, Ferreira LM, Sakka K, Knox JP, Gilbert HJ, and Fontes CM. 2015. Family 46 Carbohydrate-Binding Modules contribute to the enzymatic hydrolysis of xyloglucan and beta-1,3-1,4-glucans through distinct mechanisms. J Biol Chem 290:10572-10586

Wiegel J, Mothershed CP, and Puls J. 1985. Differences in xylan degradation by various noncellulolytic thermophilic anaerobes and Clostridium thermocellum. Appl Environ Microbiol 49:656-659.

Wilson DB, and Irwin DC. 1999. Genetics and properties of cellulases. Adv Biochem Eng 65:121.

Wilson DB, and Kostylev M. 2012. Cellulase processivity. Methods in molecular biology (Clifton, NJ) 908:93-99.

Winn MD, Isupov MN, and Murshudov GN. 2001. Use of TLS parameters to model anisotropic displacements in macromolecular refinement. Acta Crystallogr D 57:122-133.

Winn MD, Murshudov GN, and Papiz MZ. 2003. Macromolecular TLS refinement in REFMAC at moderate resolution. Methods Enzymol 374:300-321.

Yaniv O, Fichman G, Borovok I, Shoham Y, Bayer EA, Lamed R, Shimon LJ, and Frolow F. 2014. Fine-structural variance of family 3 carbohydrate-binding modules as extracellular biomass-sensing components of Clostridium thermocellum anti-sigmaI factors. Acta Crystallogr D 70:522-534.

Yaniv O, Frolow F, Levy-Assraf M, Lamed R, and Bayer EA. 2012a. Interactions between family 3 carbohydrate binding modules (CBMs) and cellulosomal linker peptides. Methods Enzymol 510:247-259.

Yaniv O, Petkun S, Shimon LJ, Bayer EA, Lamed R, and Frolow F. 2012b. A single mutation reforms the binding activity of an adhesion-deficient family 3 carbohydrate-binding module. Acta Crystallogr D 68:819-828.

Yaniv O, Shimon LJ, Bayer EA, Lamed R, and Frolow F. 2011. Scaffoldin-borne family 3b carbohydrate-binding module from the cellulosome of Bacteroides cellulosolvens: structural diversity and significance of calcium for carbohydrate binding. Acta Crystallogr D 67:506-515.

Yi Z, Su X, Revindran V, Mackie RI, and Cann I. 2013. Molecular and biochemical analyses of CbCel9A/Cel48A, a highly secreted multi-modular cellulase by Caldicellulosiruptor bescii during growth on crystalline cellulose. PloS One 8:e84172.

Zhou W, Irwin DC, Escovar-Kousen J, and Wilson DB. 2004. Kinetic studies of Thermobifida fusca Ce19A active site mutant enzymes. Biochemistry 43:9655-9663.

Zmudka MW, Thoden JB, and Holden HM. 2013. The structure of DesR from Streptomyces venezuelae, a beta-glucosidase involved in macrolide activation. Protein Sci 22:883-892.

Zverlov VV, Velikodvorskaya GA, and Schwarz WH. 2003. Two new cellulosome components encoded downstream of celI in the genome of Clostridium thermocellum: the nonprocessive endoglucanase CelN and the possibly structural protein CseP. Microbiology 149:515-524.

Peer] reviewing PDF | (2015:05:5166:1:0:NEW 29 Jun 2015) 
820 Figure captions:

821 Figure 1. Schematic diagram of the Ce19I gene product (top) and the recombinant proteins (A-

822 D) prepared for this study. The GH9 module alone (B) was prepared with and without an N-

823 terminal His tag (shown schematically in the figure), and the CBM3c's were prepared with C-

824 terminal His tags. Scale shows the number of amino acid residues and the boundaries of the

825 different regions of the protein.

826 Figure 2. Recovery of activity upon association of CBM3c (with and without linker) and GH9.

827 CMCase activity ( $\mu$ mol reducing sugar released in a 10-min reaction) of His-tagged GH9, mixed

828 either with $\mathrm{CBM} 3 \mathrm{c} L$ (diamonds) or CBM3cNL (squares), was examined. A fixed amount (70

829 pmol) of the GH9 catalytic module was mixed with increasing amounts of the indicated helper

830 module, and their respective activities were compared to that of the intact Cel9I core (GH9-

831 CBM3c, set as $100 \%)$.

832 Figure 3. Reassembled GH9-CBM3c from Cel9I. C and $\mathrm{N}$ termini are indicated, and the break

833 between the GH9 and CBM3c modules is marked by a red ellipse. A. The in vitro reassembled

834 complex of the catalytic (GH9, wheat) and carbohydrate-binding (CBM3c, green) modules of

835 Ce19I from C. thermocellum, cartoon representation. Calcium atoms are shown as magenta-

836 colored spheres. B. Stereo-view (cross-eyed) of the superposition of the reassembled GH9-

837 CBM3c structure of $C$. thermocellum Ce19I (red) with the bimodular structures of $C$.

838 cellulolyticum Ce19G (blue) and T. fusca Ce19A (green).

839 Figure 4. Structural components of the reassembled C. thermocellum GH9-CBM3c. A.

840 Structure of the GH9 catalytic module, cartoon representation. Twelve $\alpha$-helices form an $(\alpha / \alpha)_{6^{-}}$

841 barrel fold. Pairs of helices, comprising the fold, are emphasized by red, blue, yellow, magenta,

842 cyan and green. B. Surface representation of the reassembled GH9-CBM3c complex. The

843 residues are shaded according to the extent of their conservation with Ce19G from $C$.

844 cellulolyticum and Ce19A from T. fusca. Darker blue indicates higher conservation. Top, birds-

845 eye view of the catalytic cleft. Bottom, lateral view, showing the flat surface (red bar). Pink

846 ellipse indicates the catalytic cleft, and green ellipse designates terminal portion of the catalytic

847 site. C. Close-up (same orientation as in B, top) of the catalytic cleft of the Cel9I GH9 module

848 showing functional residues. Carbohydrate-binding residue carbons are colored gray, catalytic 
849 residue carbons are colored yellow. Loop 243-254 carbons are colored in light blue. D. Calcium-

850 binding site of the $C$. thermocellum Ce19I GH9 module. Coordinating residues are shown in stick

851 representation. The calcium ion is colored magenta, and distances to the coordinating atoms are

852 indicated.

853 Figure 5. Structure of the $\mathrm{CBM} 3 \mathrm{c}$ of Cel9I from C. thermocellum. $\mathrm{C}$ and $\mathrm{N}$ termini are

854 indicated A. Cartoon representation, $\beta$-strands are numbered according to the alignment with

855 Cel9G from C. cellulolyticum, and Ce19A from T. fusca. B. Calcium-binding site of the CBM3c.

856 C. Birds-eye view of the flat surface. Residues are shaded according to their degree of

857 conservation with C. cellulolyticum Ce19G and T. fusca CEL9A. Surface-exposed conserved

858 residues are shown in stick representation. D. Shallow groove of the CBM3c. Conserved surface

859 residues are shown in stick representation. The residues are colored according to the degree of

860 conservation in CBM3a, CBM3b and CBM3c modules derived from the sequences listed in the

861 Methods section.

862 Figure 6. Contact residues of the reassembled GH9-CBM3c complex. A. The GH9 module is 863 colored in brown, CBM3c in green and the linker in blue. Contact residues of the GH9, CBM3c

864 and linker are colored orange, green and blue, respectively. The contact residues between the

865 linker and the domains are described in the text. B. Alignment of the GH9 and CBM3c modules

866 of C. thermocellum Ce19I, C. cellulolyticum Ce19G, and T. fusca Ce19A (E4) cellulases. Contact

867 residues are highlighted in yellow. Only the relevant regions of the alignment are shown.

868 Residues of linker sequences are shown blue font.

869 Figure 7. Representative ITC titration of (A) GH9 and CBM3cNL (B) GH9 and CBM3cL. The 870 top panel shows the calorimetric titration and the bottom panel displays the integrated injection 871 heats corrected for control dilution heat. The solid line is the curve of the best fit used to derive 872 the binding parameters, and the fitted data describe an interaction of a one binding site model.

873 


\section{Table $\mathbf{1}$ (on next page)}

Table 1. Diffraction data of the GH9-CBM3c in vitro reassembled complex from Cel9I from $C$. thermocellum.

Values shown in parentheses are for the highest resolution cell. 
Table 1 Diffraction data of the GH9-CBM3c in vitro reassembled complex from Cel9I from C. thermocellum. Values shown in parentheses are for the highest resolution cell.

\begin{tabular}{ll}
\hline \multicolumn{1}{c}{ GH9-CBM3c } & ESRF \\
\hline Space group & $\mathrm{P}{ }_{1} 2_{1} 2_{1}$ \\
\hline Number of crystals & 1 \\
\hline Total rotation range $\left(^{\circ}\right)$ & 240 \\
\hline$a(\AA)$ & 70.39 \\
\hline$b(\AA)$ & 88.54 \\
\hline$c(\AA)$ & 106.49 \\
\hline V $\left(\AA^{3}\right)$ & 663743.40 \\
\hline Resolution range $(\AA)$ & $30-1.68(1.71-1.68)$ \\
\hline Total number of reflections & 676571 \\
\hline Unique reflections & 76727 \\
\hline Mosaicity range $\left({ }^{\circ}\right)$ & $0.18-0.46$ \\
\hline Average redundancy & 9.0 \\
\hline Completeness, overall $(\%)$ & $97.9(74.8)$ \\
\hline Mean I/ $\sigma(\mathrm{I})$ & $34.72(2.08)$ \\
\hline$R_{\text {merge }} \dagger(\%)$ & $7.4(49.8)$ \\
\hline
\end{tabular}

2

$3 \dagger R_{\text {merge }}=\Sigma_{h k l} \Sigma_{i}\left|I_{i}(h k l)-<I(h k l)>\right| \Sigma_{h k l} \Sigma_{i} I_{i}(h k l)$, where $\Sigma_{h k l}$ denotes the sum over all reflections and $\Sigma_{i}$ the sum over all 4 equivalent and symmetry-related reflections.

5

6

7

8 


\section{Table 2 (on next page)}

Table 2. Refinement statistics and results of MolProbity validation. 
Table 2 Refinement statistics and results of MolProbity validation

$\dagger$ Clash score is the number of serious steric overlaps (>0.4 $\AA$ ) per 1000 atoms.

\begin{tabular}{|c|c|}
\hline Protein & Reassembled GH9 and CBM3c (Cel9I) \\
\hline Space group & $\mathrm{P} 2{ }_{1} 2_{1} 2_{1}$ \\
\hline Resolution range & $30-1.68$ \\
\hline No. of reflections in working set & 71559 \\
\hline No. of reflections in test set & 3580 \\
\hline No. of protein atoms & 5071 \\
\hline No. of solvent atoms & 835 \\
\hline No. of $\mathrm{Cl}$ ion atoms & 3 \\
\hline No. of $\mathrm{Ca}$ ion atoms & 2 \\
\hline Overall B factor from Wilson plot $\left(\AA^{2}\right)$ & 16.06 \\
\hline Averaged B factor $\left(\AA^{2}\right)$ & 21.12 \\
\hline $\mathrm{R}_{\text {cryst }}$ & 0.1441 \\
\hline $\mathrm{R}_{\text {free }}$ & 0.1759 \\
\hline \multicolumn{2}{|l|}{ Geometry } \\
\hline RMS bonds $(\AA)$ & 0.014 \\
\hline RMS bond angles $\left({ }^{\circ}\right)$ & 1.371 \\
\hline \multicolumn{2}{|l|}{ MolProbity validation } \\
\hline Ramachandran favored (\%) (goal >98\%) & 96.7 \\
\hline Ramachandran outliers $(\%)($ goal $<0.2 \%)$ & 0.5 \\
\hline $\mathrm{C}_{\beta}$ deviations $>0.25 \AA$ (goal 0 ) & 1 \\
\hline$\dagger$ Clash score (all atoms) & 2.88 \\
\hline Rotamer outliers $(\%)($ goal $<1 \%)$ & 0.8 \\
\hline Residues with bad bonds $(\%)$ (goal $<1 \%)$ & 0.00 \\
\hline Residues with bad angles $(\%)($ goal $<0.5)$ & 0.33 \\
\hline
\end{tabular}

1 


\section{1}

Figure 1. Schematic diagram of the Cel9I gene product (top) and the recombinant proteins (A-D) prepared for this study.

The GH9 module alone (B) was prepared with and without an N-terminal His tag (shown schematically in the figure), and the CBM3c's were prepared with C-terminal His tags. Scale shows the number of amino acid residues and the boundaries of the different regions of the protein.

Ce19I gene product

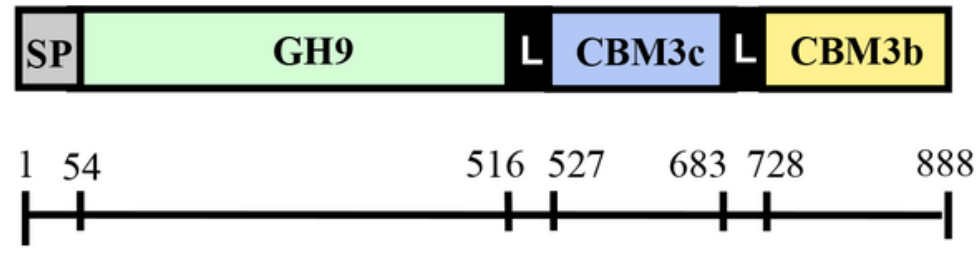

(a) Cel9I (full-length)

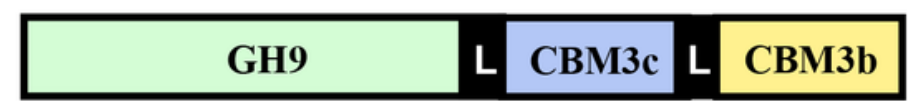

(b) GH9

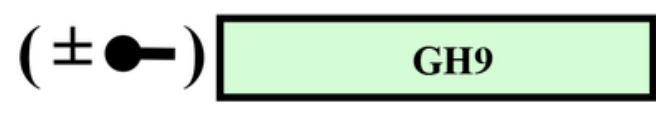

(c) $\mathrm{CBM} 3 \mathrm{c} L$ (with linker)

L CBM3c

(d) $\mathrm{CBM} 3 \mathrm{c} N L$ (no linker)

\section{CBM3c}


2

Figure 2. Recovery of activity upon association of CBM3C (with and without linker) and GH9.

CMCase activity ( $\mu \mathrm{mol}$ reducing sugar released in a 10-min reaction) of His-tagged GH9, mixed either with CBM3CL (diamonds) or CBM3CNL (squares), was examined. A fixed amount (70 pmol) of the $\mathrm{GH} 9$ catalytic module was mixed with increasing amounts of the indicated helper module, and their respective activities were compared to that of the intact Cel9l core (GH9-CBM3C, set as 100\%).

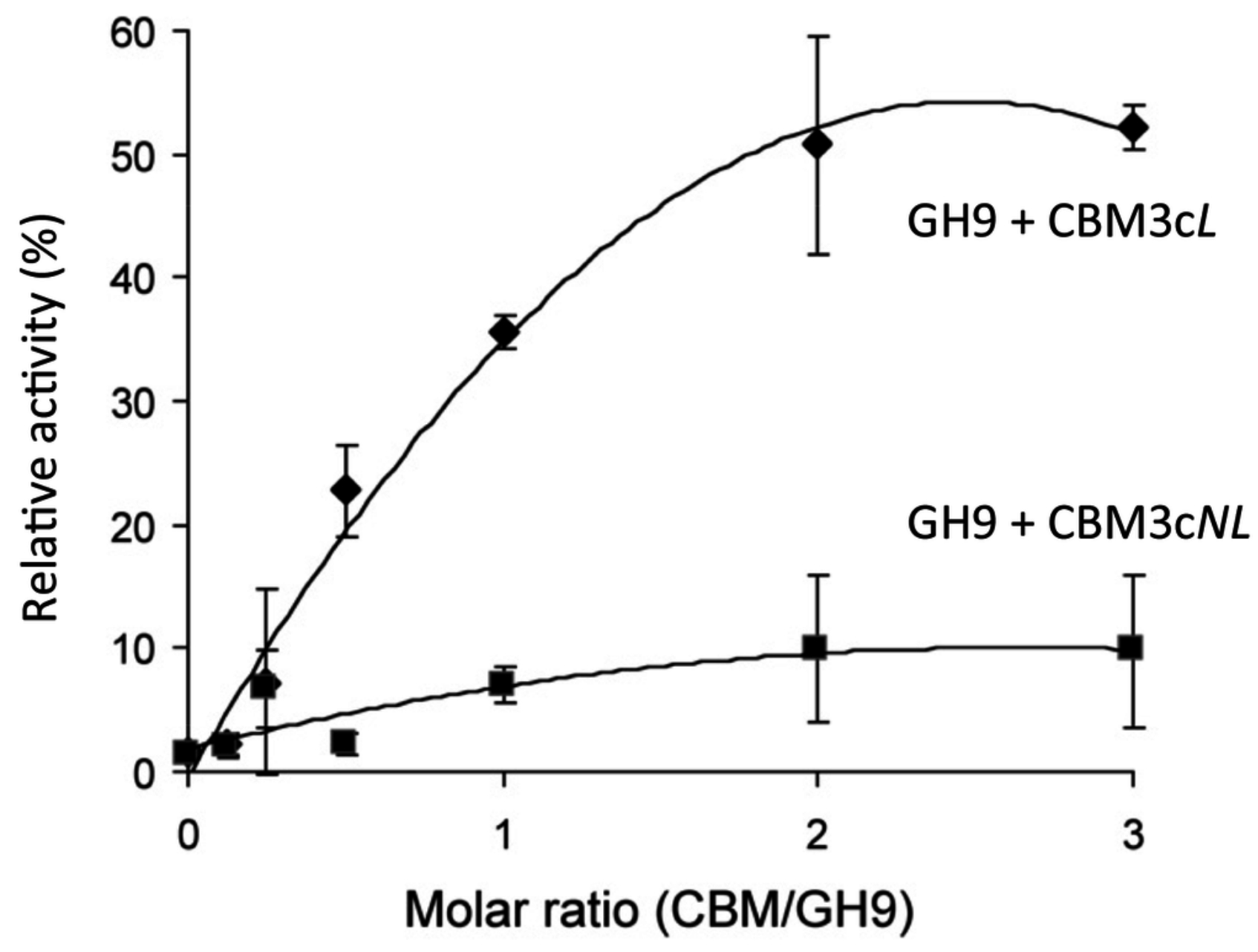




\section{3}

Figure 3. Reassembled GH9-CBM3c from Cel9I. C and N termini are indicated, and the break between the $\mathrm{GH} 9$ and $\mathrm{CBM} 3 \mathrm{C}$ modules is marked by a red ellipse.

A. The in vitro reassembled complex of the catalytic ( $\mathrm{GH} 9$, wheat) and carbohydrate-binding (CBM3c, green) modules of Cel9l from C. thermocellum, cartoon representation. Calcium atoms are shown as magenta-colored spheres. B. Stereo-view (cross-eyed) of the superposition of the reassembled GH9-CBM3c structure of $C$. thermocellum Cel9I (red) with the bimodular structures of $C$. cellulolyticum Cel9G (blue) and $T$. fusca Cel9A (green). 
A

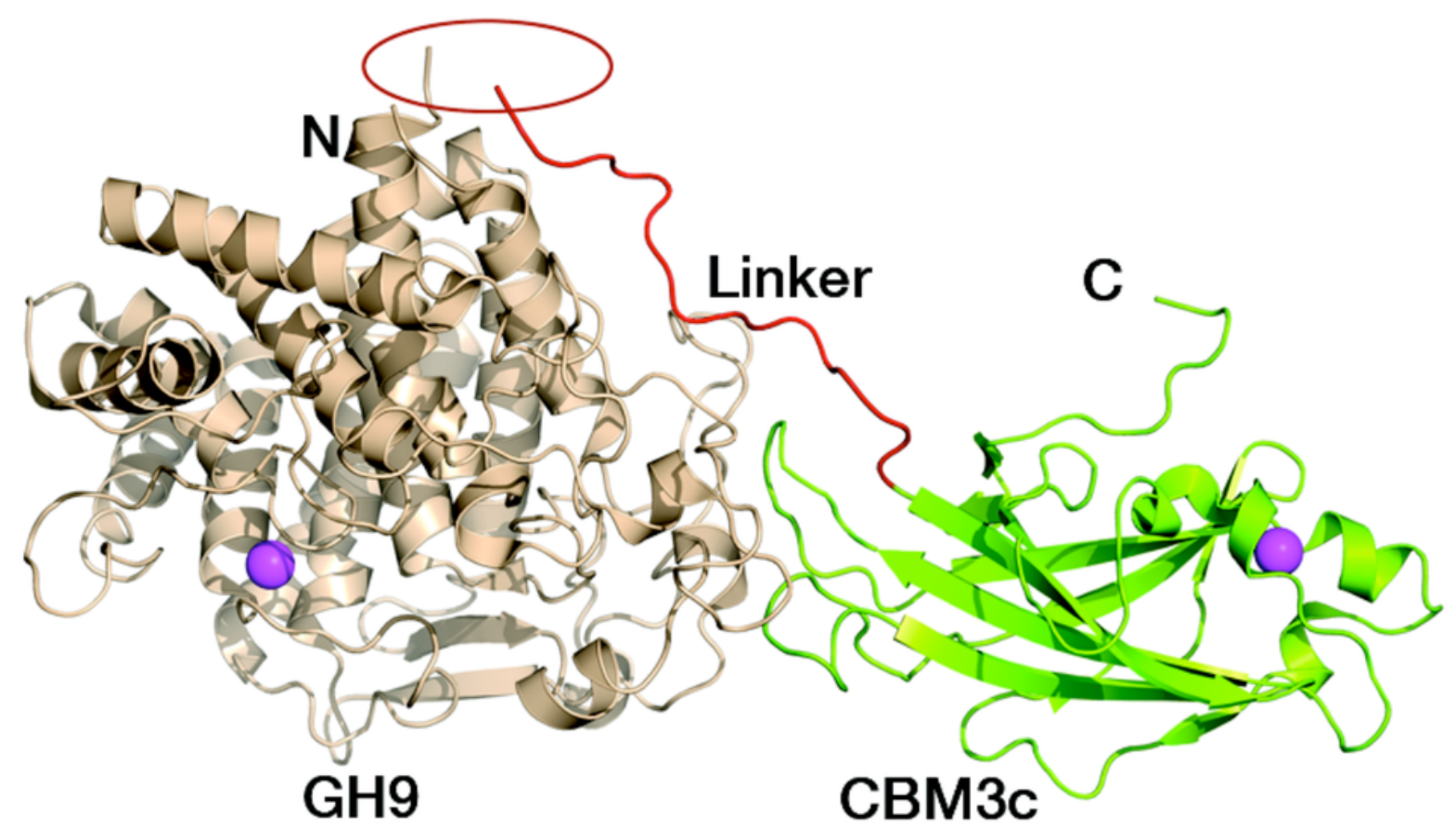

B
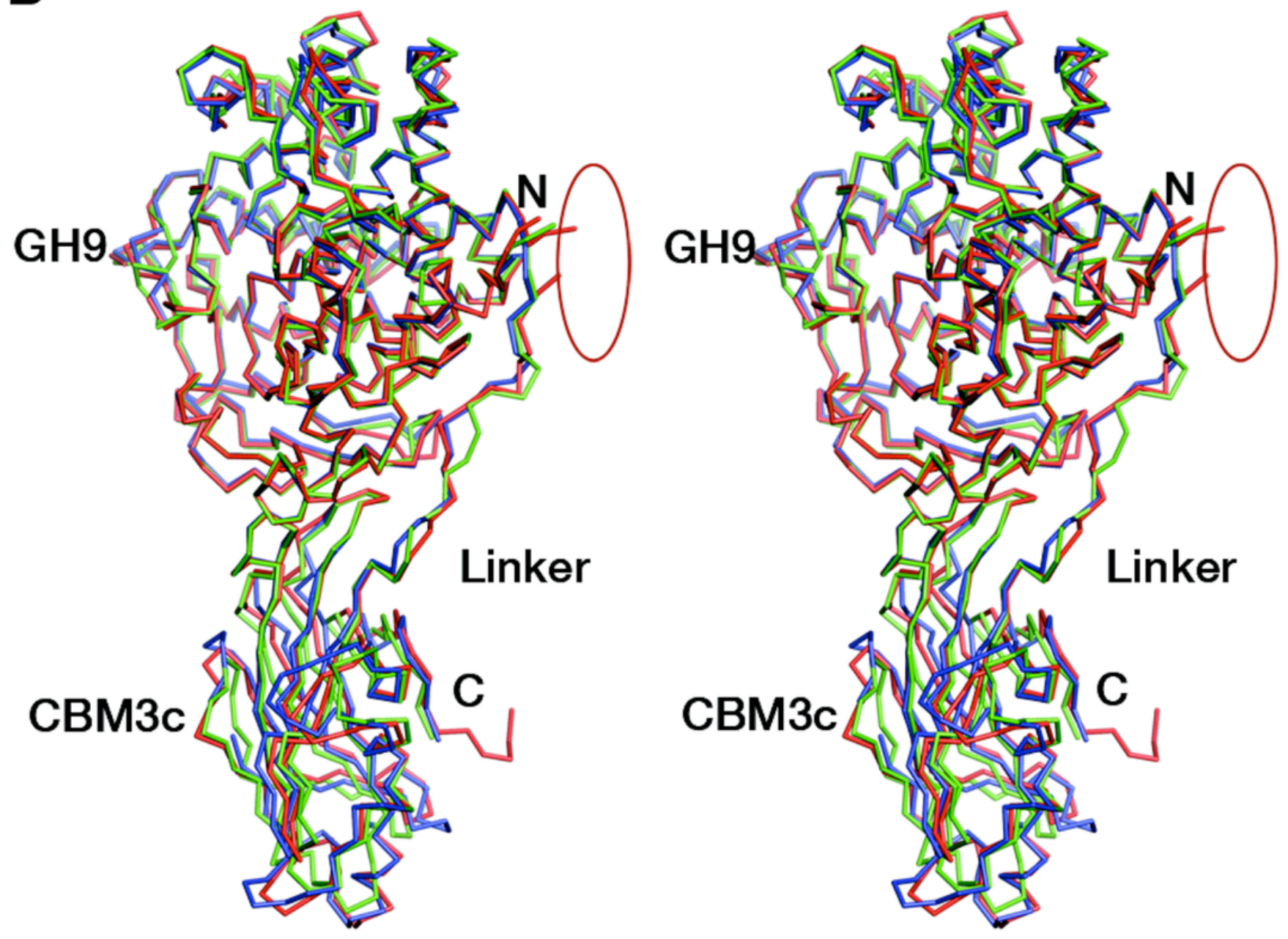


\section{4}

Figure 4. Structural components of the reassembled $C$. thermocellum GH9-CBM3c.

A. Structure of the GH9 catalytic module, cartoon representation. Twelve $\alpha$-helices form an $(\alpha / \alpha)_{6}$-barrel fold. Pairs of helices, comprising the fold, are emphasized by red, blue, yellow, magenta, cyan and green. B. Surface representation of the reassembled GH9-CBM3C complex. The residues are shaded according to the extent of their conservation with Cel9G from C. cellulolyticum and Cel9A from T. fusca. Darker blue indicates higher conservation. Top, birds-eye view of the catalytic cleft. Bottom, lateral view, showing the flat surface (red bar). Pink ellipse indicates the catalytic cleft, and green ellipse designates terminal portion of the catalytic site. C. Close-up (same orientation as in B, top) of the catalytic cleft of the Cel9I GH9 module showing functional residues. Carbohydrate-binding residue carbons are colored gray, catalytic residue carbons are colored yellow. Loop 243-254 carbons are colored in light blue. D. Calcium-binding site of the $C$. thermocellum Cel9I GH9 module. Coordinating residues are shown in stick representation. The calcium ion is colored magenta, and distances to the coordinating atoms are indicated. 
A

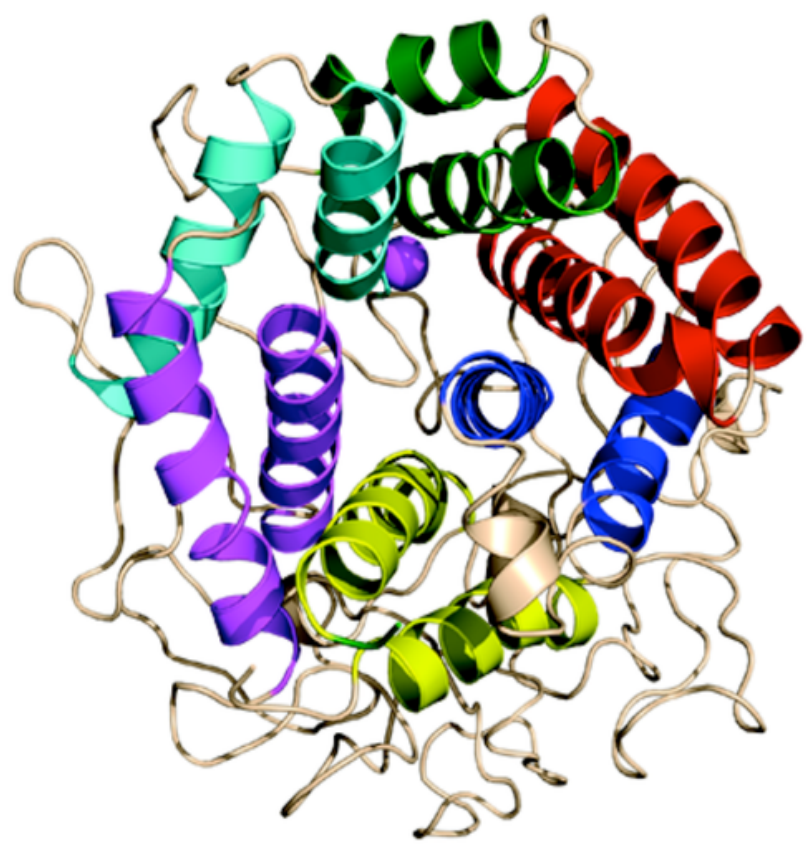

B
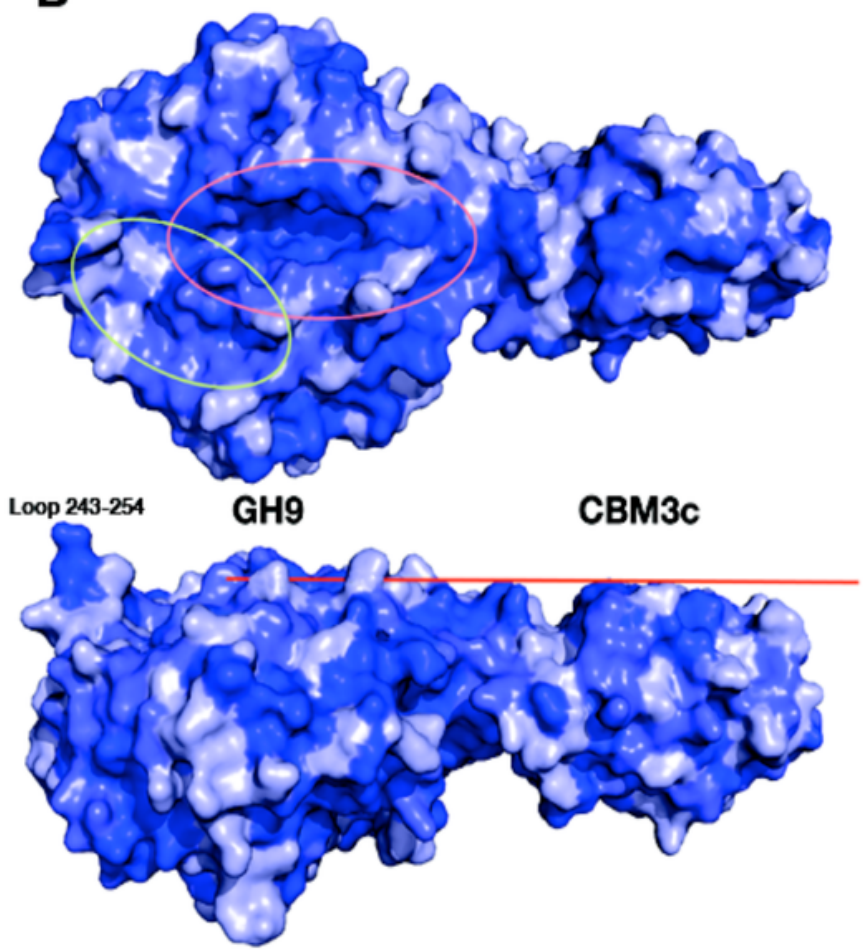

C

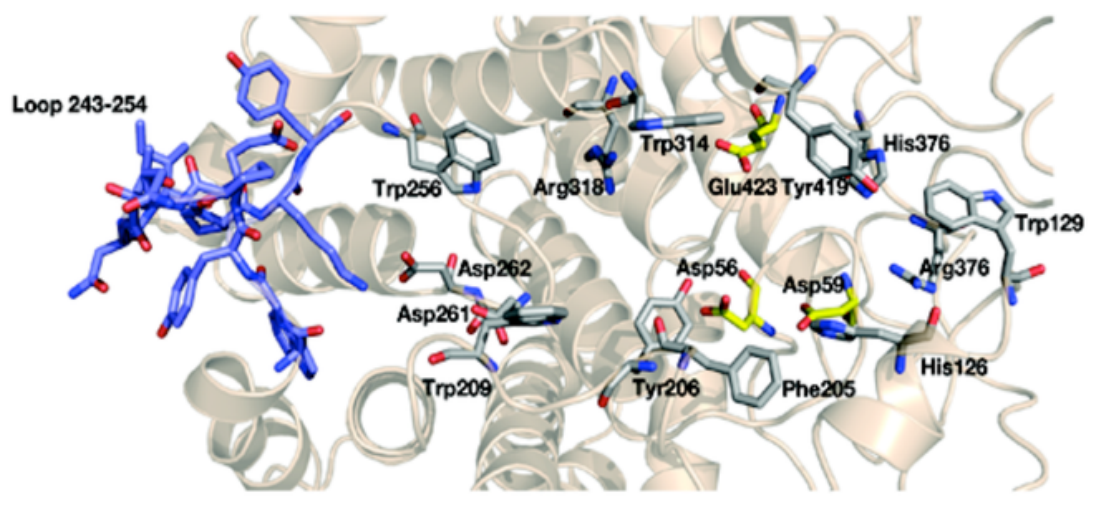

D

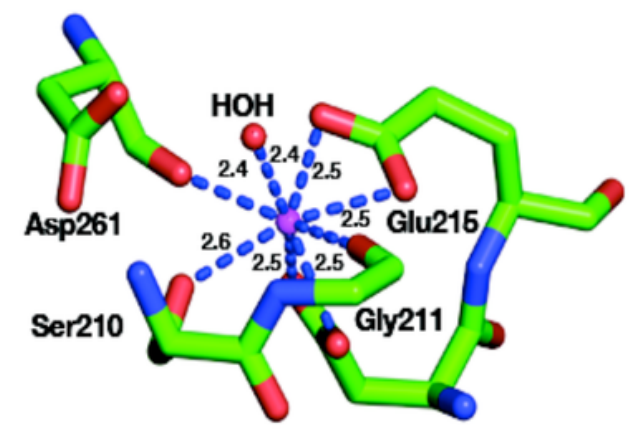




\section{5}

Figure 5. Structure of the CBM3c of Cel9I from $C$. thermocellum.

$\mathrm{C}$ and $\mathrm{N}$ termini are indicated $\mathbf{A}$. Cartoon representation, $\beta$-strands are numbered according to the alignment with Cel9G from C. cellulolyticum, and Cel9A from T. fusca. B. Calciumbinding site of the CBM3c. C. Birds-eye view of the flat surface. Residues are shaded according to their degree of conservation with C. cellulolyticum Cel9G and T. fusca CEL9A. Surface-exposed conserved residues are shown in stick representation. D. Shallow groove of the CBM3c. Conserved surface residues are shown in stick representation. The residues are colored according to the degree of conservation in CBM3a, CBM3b and CBM3c modules derived from the sequences listed in the Methods section.

A
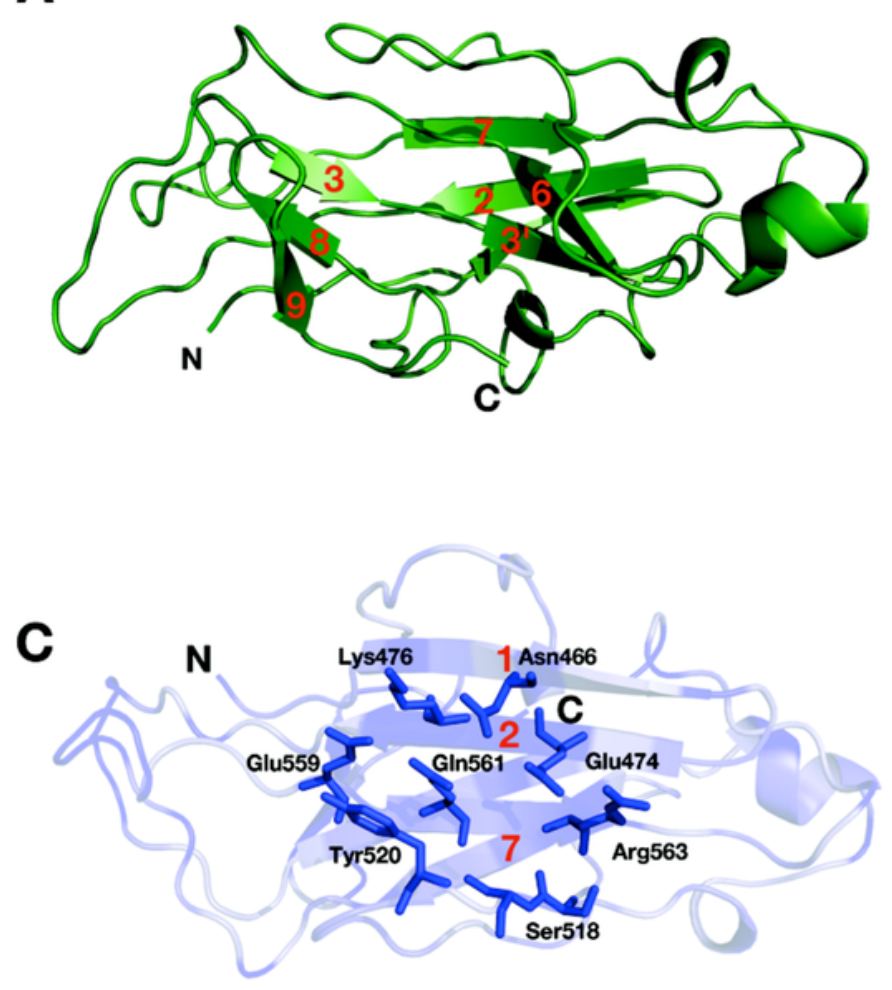

B
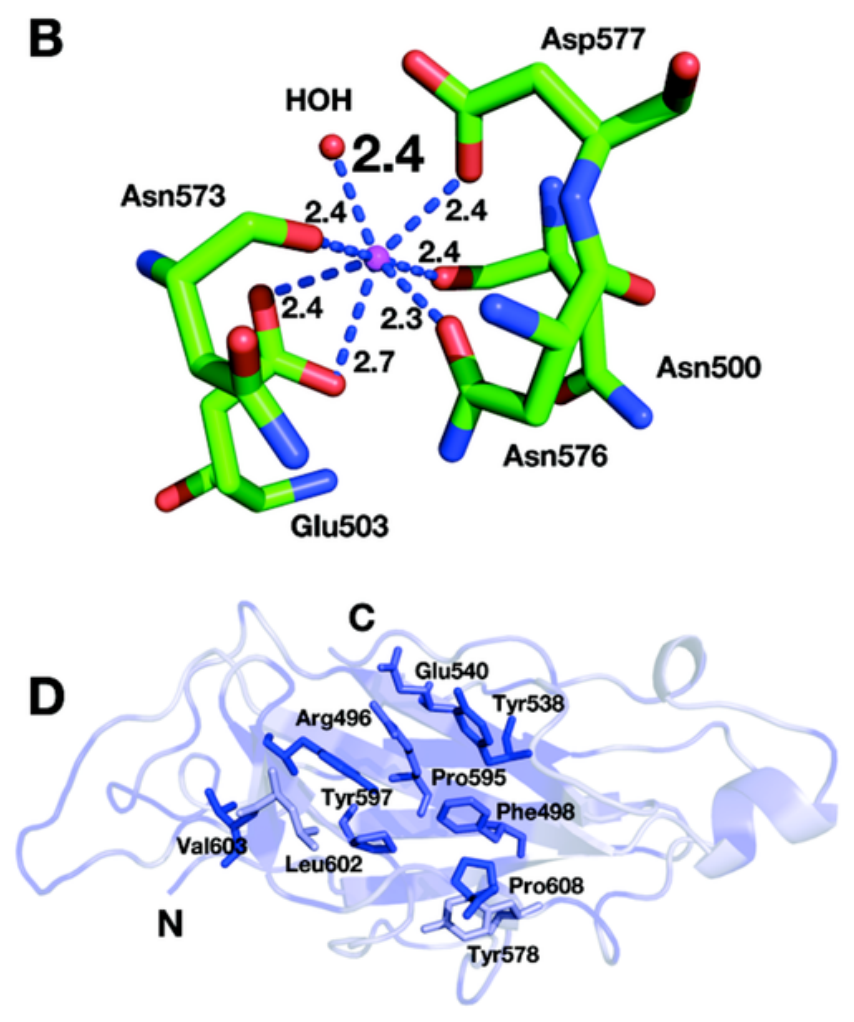


\section{6}

Figure 6. Contact residues of the reassembled GH9-CBM3c complex.

A. The GH9 module is colored in brown, $\mathrm{CBM} 3 \mathrm{C}$ in green and the linker in blue. Contact residues of the GH9, CBM3C and linker are colored orange, green and blue, respectively. The contact residues between the linker and the domains are described in the text. B. Alignment of the GH9 and CBM3c modules of $C$. thermocellum Cel9I, C. cellulolyticum Cel9G, and $T$. fusca Cel9A (E4) cellulases. Contact residues are highlighted in yellow. Only the relevant regions of the alignment are shown. Residues of linker sequences are shown blue font. 


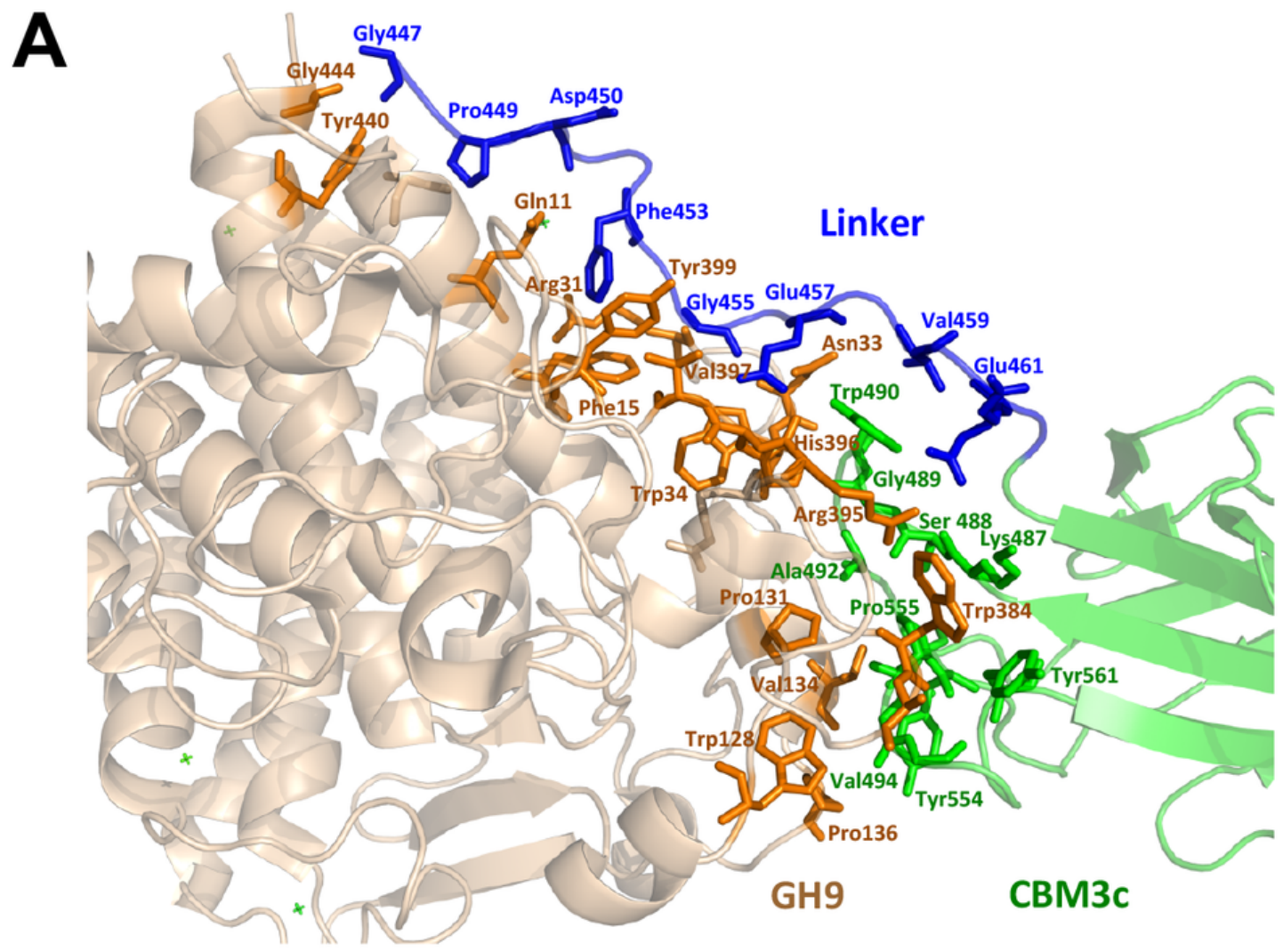

\section{GH9 module}

CelI

CelG

E4

CelI

CelG

E4

CelI

CelG

$\mathrm{E} 4$

CelI

Celg

E4

TGAFNYGEALQKAIFFYECQRSGKLDSSTLRLNWRGDSGLDDGKDAGIDLTGGWYDAGDH 60 AGTYNYGEALQKSIMFYEFQRSGDLPAD-KRDNWRDDSGMKDGSDVGVDLTGGWYDAGDH 59 EPAFNYAEALQKSMFFYEAQRSGKLPEN-NRVSWRGDSGLNDGADVGLDLTGGWYDAGDH 59

DGHADHAWWGPAEVMPMERPSYKVDRSSPGSTVVAETSAALAIASIIFKKVDGEYSKECL 180 DGGKDHSWWGPAEVMOMERPSFKVDASKPGSAVCASTAASLASAAVVFKSSDPTYAEKCI 179 DGDADHKWWGPAEVMPMERPSFKVDPSCPGSDVAAETAAAMAASSIVFADDDPAYAATLV 179

G--RSFVVGFGENPPKRPHHRTAHGSWADSQMEPPEHRHVLYGALVGGPDST-DNYTDDI 416 G--RSFVVGYGVNPPQHPHHRTAHGSWTDQMTSPTYHRHTIYGALVGGPDNA-DGYTDEI 413 PRNSSYVVGFGNNPPRNPHHRTAHGSWTDSIASPAENRHVLYGALVGGPGSPNDAYTDDR 417

SNYTCNEVACDYNAGFVGLLAKMYKLYGEL 446 NNYVNNEIACDYNAGFTGALAKMYKHSG - 441 QDYVANEVATDYNAGFSSALAMLVEEYG - 445

\section{CBM3c module}

Linker

CelI GSPDPKFNGIEEVPEDEIFVEAGVNASGNNFIEIKAIVNNKSGWPARVCENLSFRYFINI 506 CelG GDPIPNFKAIEKITNDEVIIKAGLNSTGPNYTEIKAVVYNQTGWPARVTDKISFKYFMDL 501 E4 GTPLADFPPTEEPDGPEIFVEAQINTPGTTFTEIKAMIRNQSGWPARMLDKGTFRYWFTL 505

CelI EEIVNAGKSASDLQVSSSYNQGAKLS--DV--KHYKDNIYYVEVDLSGTKIYPGGQSAYK 562 CelG SEIVAAGIDPLSLVTSSNYSEGKNTKVSGVLPWDVSNNVYYVNVDLTGENIYPGGQSACR 561 E4 DE----GVDPADITVSSAYNQCATPE--D--VHHVSGDLYYVEIDCTGEKIFPGGQSEHR 557

CelI KEVQFRISAPEGTV-FNPENDYSYQGLSAGTV-VKSEYIPVYDAGVLVFGREPLE 615 Celc REVOFRTADPOGTTYWNPKNDFSYDGLPTTSTVNTVTNIPVYDNGVKVFGNEP-- 614 Peer reviewing 
7

Figure 7. Representative ITC titration of (A) GH9 and CBM3CNL (B) GH9 and CBM3CL.

The top panel shows the calorimetric titration and the bottom panel displays the integrated injection heats corrected for control dilution heat. The solid line is the curve of the best fit used to derive the binding parameters, and the fitted data describe an interaction of a one binding site model.

A

Time ( $\min )$

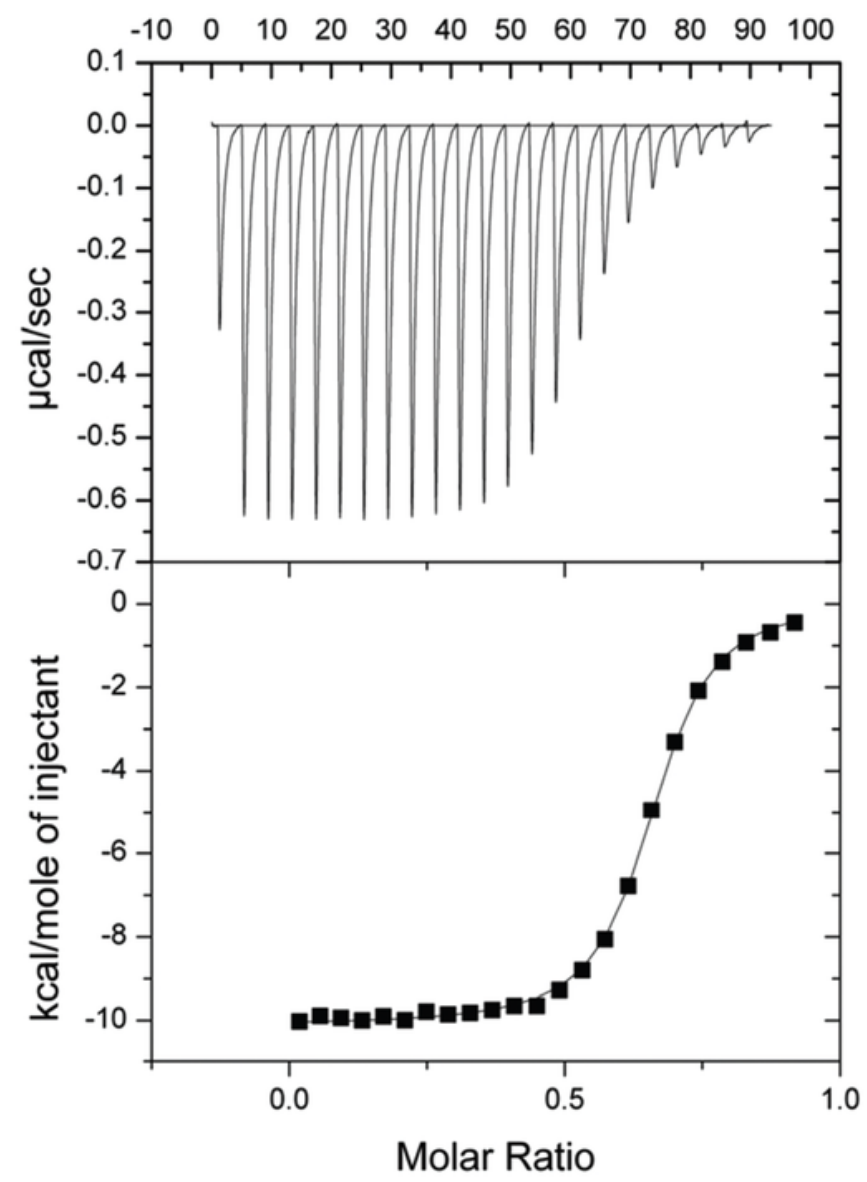

B

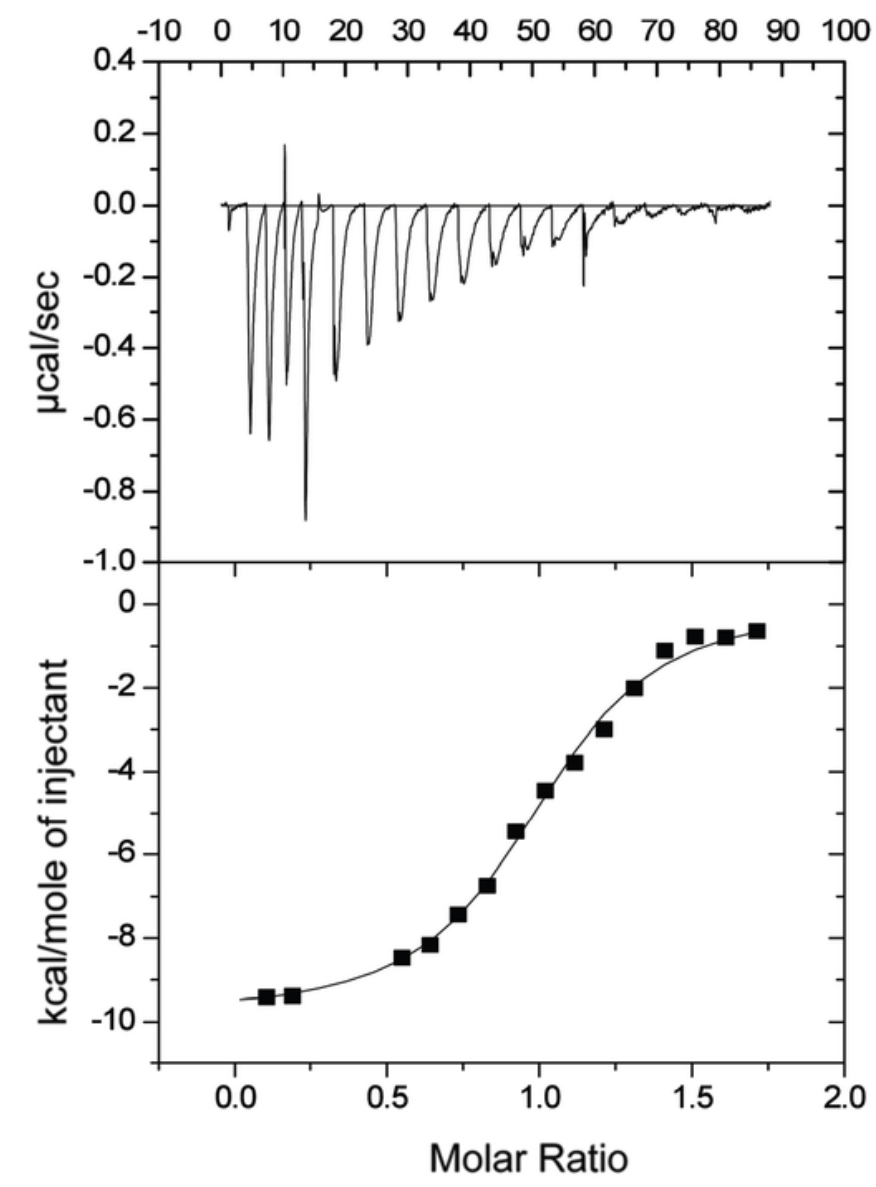

